\title{
CHEMICAL ABUNDANCES IN FIELD RED GIANTS FROM HIGH-RESOLUTION $H$-BAND SPECTRA USING THE APOGEE SPECTRAL LINELIST
}

\author{
Verne V. Smith ${ }^{1,2}$, Katia Cunha ${ }^{1,2,3}$, Matthew D. Shetrone ${ }^{4}$, Szabolcs Meszaros ${ }^{5,6}$, Carlos Allende Prieto ${ }^{5,6}$, \\ Dmitry Bizyaev ${ }^{7,8}$, Ana Garcìa PèreZ ${ }^{9}$, Steven R. Majewski ${ }^{9}$, Ricardo Schiavon ${ }^{10}$, \\ JON HOLTZMAN ${ }^{11}$, AND JeNNIFER A. JOHNSON ${ }^{12}$ \\ ${ }^{1}$ National Optical Astronomy Observatories, Tucson, AZ 85719, USA; vsmith@noao.edu \\ ${ }^{2}$ Observatorio Nacional, Sao Cristovao, Rio de Janeiro, Brazil \\ ${ }^{3}$ Astronomy Department \& Steward Observatory, University of Arizona, Tucson, AZ 85721 \\ ${ }^{4}$ Department of Astronomy and McDonald Observatory, University of Texas, Austin, TX 78712, USA \\ ${ }^{5}$ Instituto d'Astrofisica de Canarias, E-38205, La Laguna, Tenerife, Spain \\ ${ }^{6}$ Departmento de Astrofisica, Universidad de La Laguna, 38206 La Laguna, Tenerife, Spain \\ ${ }^{7}$ Apache Point Observatory, Sunspot, NM 88349, USA \\ ${ }^{8}$ Strenberg Astronomical Institute, Moscow 119992, Russia \\ ${ }^{9}$ Department of Astronomy, University of Virginia, Charlottesville, VA 22904, USA \\ ${ }^{10}$ Astrophysics Research Institute, Liverpool John Moores University, Liverpool L3 5UX, UK \\ ${ }^{11}$ Department of Astronomy, New Mexico State University, Las Cruces, NM 88003, USA \\ ${ }^{12}$ Department of Astronomy, Ohio State University, Columbus, OH 43210, USA \\ Received 2012 October 12; accepted 2012 December 17; published 2013 February 13
}

\begin{abstract}
High-resolution $H$-band spectra of five bright field $\mathrm{K}, \mathrm{M}$, and MS giants, obtained from the archives of the Kitt Peak National Observatory Fourier transform spectrometer, are analyzed to determine chemical abundances of 16 elements. The abundances were derived via spectrum synthesis using the detailed linelist prepared for the Sloan Digital Sky Survey III Apache Point Galactic Evolution Experiment (APOGEE), which is a high-resolution near-infrared spectroscopic survey to derive detailed chemical abundance distributions and precise radial velocities for 100,000 red giants sampling all Galactic stellar populations. The red giant sample studied here was chosen to probe which chemical elements can be derived reliably from the $H$-band APOGEE spectral region. These red giants consist of two K-giants ( $\alpha$ Boo and $\mu$ Leo), two M-giants ( $\beta$ And and $\delta$ Oph), and one thermally pulsing asymptotic giant branch (TP-AGB) star of spectral type MS (HD 199799). Measured chemical abundances include the cosmochemically important isotopes ${ }^{12} \mathrm{C},{ }^{13} \mathrm{C},{ }^{14} \mathrm{~N}$, and ${ }^{16} \mathrm{O}$, along with $\mathrm{Mg}, \mathrm{Al}, \mathrm{Si}, \mathrm{K}, \mathrm{Ca}, \mathrm{Ti}, \mathrm{V}, \mathrm{Cr}, \mathrm{Mn}, \mathrm{Fe}$, $\mathrm{Co}, \mathrm{Ni}$, and $\mathrm{Cu}$. The $\mathrm{K}$ and $\mathrm{M}$ giants exhibit the abundance signature of the first dredge-up of $\mathrm{CN}$-cycle material, while the TP-AGB star shows clear evidence of the addition of ${ }^{12} \mathrm{C}$ synthesized during ${ }^{4} \mathrm{He}$-burning thermal pulses and subsequent third dredge-up. A comparison of the abundances derived here with published values for these stars reveals consistent results to $\sim 0.1$ dex. The APOGEE spectral region and linelist is thus well suited for probing both Galactic chemical evolution, as well as internal nucleosynthesis and mixing in populations of red giants via high-resolution spectroscopy.
\end{abstract}

Key words: stars: abundances - stars: late-type

Online-only material: color figures

\section{INTRODUCTION}

The Apache Point Observatory Galactic Evolution Experiment (APOGEE) is one of four experiments that are part of the Sloan Digital Sky Survey III (SDSS-III; Eisenstein et al. 2011). APOGEE is obtaining high-resolution $(R \sim 22,300)$, high signal-to-noise ratio ( $\mathrm{S} / \mathrm{N} \geqslant 100$ per pixel), $H$-band $(\lambda 1.51-1.69 \mu \mathrm{m})$ spectra of evolved, late-type stars, with the goal being to measure chemical abundances of 15 elements per star. Coupled with radial velocities that are accurate to $\sim 100 \mathrm{~m} \mathrm{~s}^{-1}$, APOGEE is creating the first high-precision spectroscopic and radial-velocity survey of all Galactic stellar populations (bulge, bar, disks, halo) using a uniform set of stellar tracers and spectral diagnostics, with a plan to observe and derive these parameters for 100,000 red giants by the end of 2014 .

The physical and chemical parameters of the APOGEE stars are derived from a suite of software packages that, together, are called the APOGEE Stellar Parameters and Chemical Abundances Pipeline (ASPCAP); the details of this software and its analysis techniques will be discussed by $\mathrm{A}$. Garcìa Pèrez et al. (in preparation). The first-generation output of ASPCAP, which will be based on one-dimensional (1-D) stellar atmosphere analyses in LTE, will consist of the stellar parameters of effective temperature, $T_{\text {eff }}$, surface gravity (noted as $\log g$ ), and the 1-D microturbulence parameter $(\xi)$, along with the abundances of up to 16 elements $\left(\mathrm{Fe},{ }^{12} \mathrm{C},{ }^{14} \mathrm{~N},{ }^{16} \mathrm{O}, \mathrm{Mg}, \mathrm{Al}, \mathrm{Si}, \mathrm{K}, \mathrm{Ca}, \mathrm{Ti}\right.$, $\mathrm{V}, \mathrm{Cr}, \mathrm{Mn}, \mathrm{Co}, \mathrm{Ni}$, or $\mathrm{Cu}$ ) and, for many stars, the ${ }^{13} \mathrm{C}$ isotopic abundance.

One of the central components of the ASPCAP machinery is a spectral linelist constructed to produce the synthetic spectra that are used to compare to the observed ones; the APOGEE linelist will be presented and discussed in detail by M. Shetrone et al. (in preparation).

The goal of this study is to analyze a small number nearby field red giants with well-defined stellar parameters and use the APOGEE linelist to derive detailed chemical abundances via standard "manual" abundance analysis techniques (Ramirez \& Allende Prieto 2011 is a recent example using such techniques). The derived stellar parameters and chemical abundances can be compared to the same quantities derived via ASPCAP using the same spectra, linelist, and model atmosphere grid. This comparison will be an important test of ASPCAP. This work will also present new abundances in these stars based on an extensive and up-to-date spectral linelist in the near-IR $H$ band. 


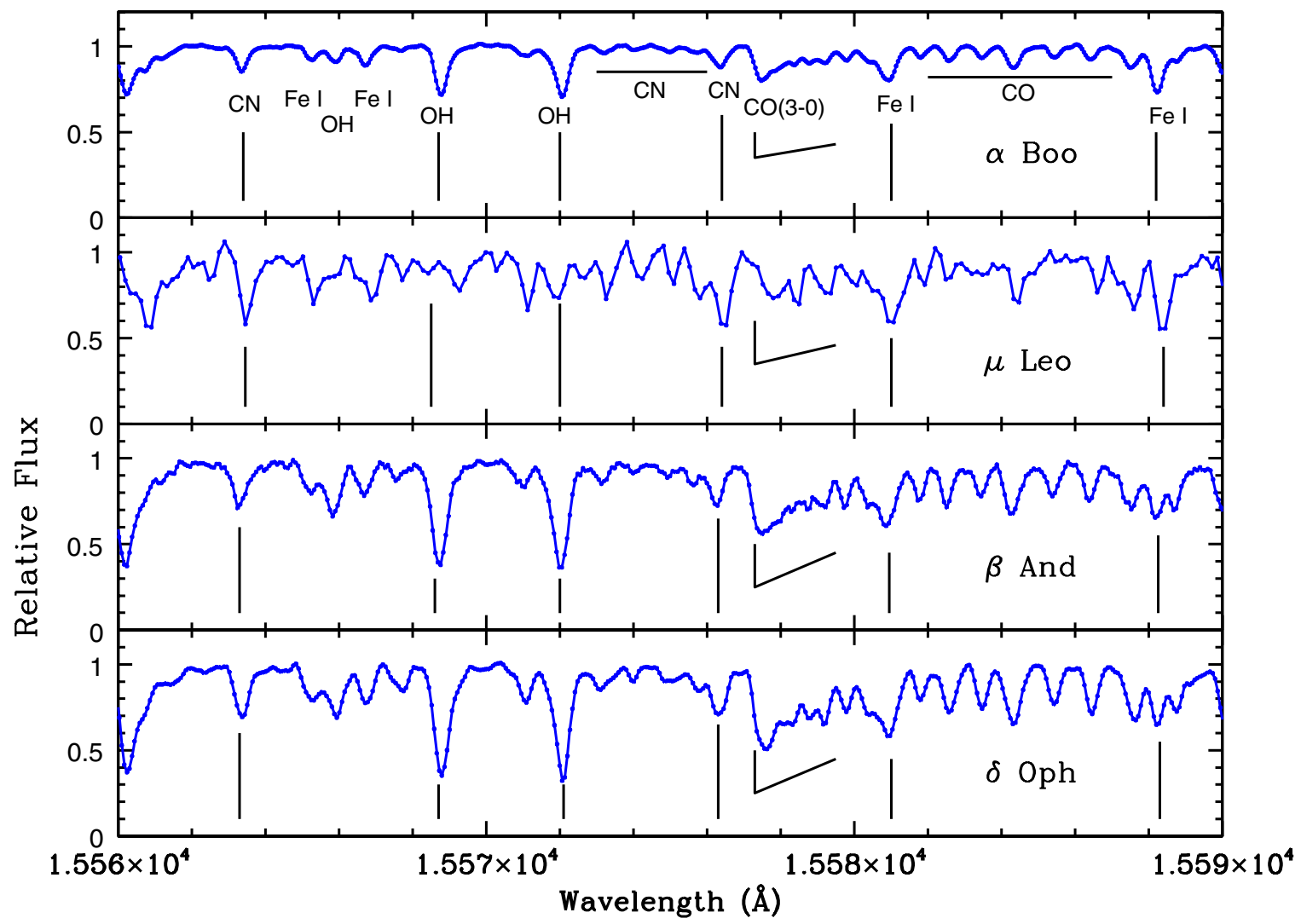

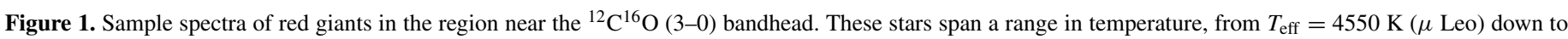

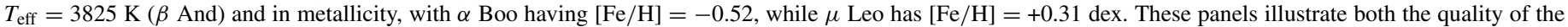
spectra and the variation of molecular and atomic line absorption with primarily $T_{\text {eff }}$.

(A color version of this figure is available in the online journal.)

Abundances will be presented in this paper using two different types of nomenclature, with one abundance scale defined as $A(\mathrm{X})=\log (N(\mathrm{X}) / N(\mathrm{H}))+12.0$. The other scale compares the element ratio of $N(\mathrm{X}) / N(\mathrm{Q})$ in a target star to the Sun via $[\mathrm{X} / \mathrm{Q}]=\left(A(\mathrm{X})_{\text {TargetStar }}-A(\mathrm{X})_{\odot}\right)-\left(A(\mathrm{Q})_{\text {TargetStar }}-A(\mathrm{Q})_{\odot}\right)$.

\section{OBSERVATIONAL DATA: THE FOURIER TRANSFORM SPECTRA OF BRIGHT FIELD RED GIANTS}

The APOGEE high-resolution spectra cover the wavelength range from $\sim 15100$ to $16900 \AA$ and to analyze bright red giants over this spectral interval, data were obtained from the archives of the Fourier transform spectrometer (FTS; Hall et al. 1979) that operated at the coude focus of the Kitt Peak National Observatory Mayall $4 \mathrm{~m}$ reflector. A more complete description of observing techniques and the original data is discussed in Hinkle et al. (1995), in particular for the spectral atlas of $\alpha$ Boo.

The originally reduced spectra of selected stars were recorded as flux versus wavenumber $\left(\mathrm{cm}^{-1}\right)$ over different dates and runs and covered a broad region in the $H$ band. The spectra analyzed here were resampled in wavelength space and restricted to a wavelength range of $15000-17000 \AA$. The resolution of the spectra for different stars varies from $R=45,000$ to 100,000 , but were all higher than what is being observed for APOGEE. The higher resolution allows for a detailed investigation of the limits of what can be derived high-quality (i.e., high-resolution and high signal-to-noise) red giant spectra when using the APOGEE linelist.

\section{THE "STANDARD STAR" PARAMETERS}

\subsection{The Choices}

There are five stars analyzed here: the well-studied $\alpha$ Boo, a mildly metal-poor K1.5 giant, $\mu$ Leo, the prototype metalrich star (Spinrad \& Taylor 1969), which is a K1 giant, two near-solar-metallicity $\mathrm{M}$ giants, $\beta$ And (M0III) and $\delta$ Oph (M0III), and HD199799, an asymptotic giant branch (AGB) star of spectral type MS with a mild enhancement of ${ }^{12} \mathrm{C}$ and a slightly elevated ratio of $\mathrm{C} / \mathrm{O}$ (relative to solar) due to third dredge-up (Smith \& Lambert 1990). Figure 1 illustrates a small portion of the APOGEE wavelength coverage for four of the red giants here. This region from 15560 to $15590 \AA$ includes representative species and how they vary with spectral type (or effective temperature) and metallicity. Lines from the main CNO-containing molecules, $\mathrm{CO}, \mathrm{OH}$, and $\mathrm{CN}$ fall within this region, as noted in the figure. Of particular interest is the metalrich K-giant $\mu$ Leo, which exhibits quite strong $\mathrm{CN}$ lines; much of the variation in the spectrum of $\mu$ Leo compared to the other giants result from the high line density of $\mathrm{CN}$. This figure also points to the desirability of analyzing large numbers of red giants via spectrum synthesis techniques using a detailed linelist.

With a significant fraction of its red giant targets in the thin and thick disks, plus the inner bulge and bar, the five types of red giants studied here span a range of temperature and metallicity that provides a good test for ASPCAP. The APOGEE survey targets red giants with effective temperatures from $T_{\text {eff }}=3400 \mathrm{~K}-5000 \mathrm{~K}$, having surface gravities from 
Table 1

Red Giant Standard Stars: Observed Properties

\begin{tabular}{lrcrcrr}
\hline \hline Star & HR & SpT & $\begin{array}{c}\pi \\
(\mathrm{mas})^{\mathrm{a}}\end{array}$ & \multicolumn{1}{c}{$\begin{array}{c}d \\
(\mathrm{pc})\end{array}$} & $J-K_{\mathrm{S}}{ }^{\mathrm{b}}$ & \multicolumn{1}{c}{$M_{\text {bol }}{ }^{\mathrm{c}}$} \\
\hline$\beta$ And & 337 & M0III & $16.4 \pm 0.8$ & $61 \pm 3$ & 0.98 & $-3.06 \pm 0.11$ \\
$\mu$ Leo & 3905 & K2III & $26.3 \pm 0.2$ & $38 \pm 1$ & 0.68 & $0.41 \pm 0.05$ \\
$\alpha$ Boo & 5340 & K2III & $88.8 \pm 0.5$ & $11.3 \pm 0.1$ & 0.79 & $-0.92 \pm 0.03$ \\
$\delta$ Oph & 6056 & M0III & $19.1 \pm 0.2$ & $52.5 \pm 0.5$ & 0.97 & $-2.16 \pm 0.05$ \\
HD 199799 & $\ldots$ & M2S & $2.6 \pm 0.6$ & $386 \pm 92$ & 1.25 & $-3.64 \pm 0.54$ \\
\hline
\end{tabular}

Notes.

a Parallax from van Leeuwen (2007)

b Johnson (1966) transformed to the 2MASS system using Carpenter (2001).

${ }^{c}$ Calculated from $M_{K}$ with bolometric corrections from Bessell et al. (1998).

$\log g=3.0$ to -0.5 , and metallicities from $[\mathrm{Fe} / \mathrm{H}]=-5.0$ to +1.0 . These nearby giants have well-measured photometry and parallaxes, so temperatures, luminosities, and masses are all rather well constrained. In addition, four of the five stars have previous abundance analyses in the literature and thus provide comparison tests for the abundances derived from the APOGEE linelist.

\subsection{Basic Data}

Deriving stellar chemical abundances from high-resolution spectra requires as basic input the effective temperature $\left(T_{\text {eff }}\right)$, surface gravity (characterized as $\log g$ with $g$ as $\mathrm{cm} \mathrm{s}^{-2}$ ), and overall metallicity as the fundamental parameters of the model atmosphere. In addition, high-resolution abundance analyses of cool stars that use static 1-D stellar atmospheres also require the derivation of a non-thermal Doppler-like broadening term called microturbulence $(\xi)$, which is determined as part of the spectral analysis. The first step is the determination of $T_{\text {eff }}$ and, for these relatively nearby red giants, is set by the near-infrared magnitudes of $J$ and $K$. This particular choice is used because the APOGEE targets are selected from the Two Micron All Sky Survey (2MASS) catalog (Skrutskie et al. 2006) and therefore all have $(J-K)$ available. The aim is to focus on utilizing near-IR data and then compare to published literature results.

All of the red giants here have parallaxes measured by Hipparcos and thus have distances known to various levels of accuracy. The parallax is used to set the distance and thus $M_{K}$. Bolometric corrections from Bessell et al. (1998) are used in conjunction with $(J-K)$ to determine $M_{\text {bol }}$ and consequently stellar luminosity. Basic data for the red giants are presented in Table 1.

The most important derived quantity affecting the overall shape of the spectrum is $T_{\text {eff }}$, which here is based on an average of two $T_{\text {eff }}-(J-K)$ calibrations; one from Gonzalez Hernandez $\&$ Bonifacio (2009) and the other from Bessell et al. (1998). The Gonzalez Hernandez \& Bonifacio calibration is defined in the 2MASS photometric system of $\left(J-K_{S}\right)$, so the original Johnson (1966) $(J-K)$ colors and $K$ magnitudes for these bright red giants (which have saturated magnitudes in the 2MASS catalog) were transformed to 2MASS values using the prescription from Carpenter (2001). The differences between the calibrations of Bessell et al. (1998) and Gonzalez Hernandez \& Bonifacio (2009), for a given metallicity, are less than 30-50 K for $T_{\text {eff }} \leqslant 4500 \mathrm{~K}$, but above this temperature the Bessell et al. (1998) becomes cooler by about $100 \mathrm{~K}$ for a given $\left(J-K_{\mathrm{S}}\right)$ (where we have transformed the Bessell et al. scale onto the 2MASS system). There is also a small metallicity effect on the $\left(J-K_{\mathrm{S}}\right)$ calibration which, for a given $\left(J-K_{\mathrm{S}}\right)$, can be $\sim 100 \mathrm{~K}$ over the broad metallicity range of $[\mathrm{m} / \mathrm{H}]=-2.0$ to +0.0 (where $\mathrm{m}$ is taken to represent the generic abundance of metals, i.e., those elements other than $\mathrm{H}$ and $\mathrm{He}$ ). Given the differences between the two independent $T_{\text {eff }}$ calibrations of less than $30-50 \mathrm{~K}$ over the temperature range of the target stars, as well as their modest range in metallicity, and well-determined magnitudes with $\sim 0.02$ uncertainty in $\left(J-K_{\mathrm{S}}\right)$ (which translates to $\left.\Delta T_{\text {eff }}=30 \mathrm{~K}\right)$, the derived values of $T_{\text {eff }}$ have uncertainties of $\pm 50 \mathrm{~K}$ or less.

Given the stellar luminosity and effective temperature, these fundamental properties are then compared to stellar evolution models presented in Bertelli et al. (2008, 2009; with data from these papers taken from the Web site http://stev.oapd.inaf.it/YZVAR) to estimate stellar masses and, ultimately, surface gravities through

$$
g / g_{\odot}=\left(M / M_{\odot}\right) \times\left(L_{\odot} / L\right) \times\left(T_{\text {eff }} / T_{\text {eff } \odot}\right)^{4} .
$$

Determining the surface gravity from evolutionary tracks is an iterative process because it requires knowledge of the stellar metallicity. This is illustrated in Figure 2 where the stellar luminosity and $T_{\text {eff }}$ are plotted along with evolutionary models from a range of masses, with each panel having a different metallicity: $Z=0.017$ (top panel), 0.008 (middle panel), and 0.004 (bottom panel), corresponding to overall metallicities of $[\mathrm{m} / \mathrm{H}]=+0.07,-0.26$, and -0.56 , respectively, when taking $Z=0.0145$ for the Sun, as suggested by Lodders (2010). The illustrated evolutionary tracks follow stellar evolution up to the tip of the first-ascent red giant branch (which we label RGB). Initial model metallicities for the stars studied here are based on previously published results, with $\beta$ And, $\delta$ Oph, and HD199799 started with solar metallicity, $\alpha$ Boo with $[\mathrm{Fe} / \mathrm{H}]=$ -0.5 , and $\mu$ Leo with $[\mathrm{Fe} / \mathrm{H}]=+0.3$. As there are numerous $\mathrm{Fe}$ I lines in this spectral region, a sample of $\mathrm{Fe}$ I lines are used to determine the iron abundance. Given the limited range of metallicity spanned by the nearby red giants studied here, the Fe abundance is a good proxy for the overall stellar metallicity. If the derived $\mathrm{Fe}$ abundance differed by more than 0.1 dex from the assumed value, a new surface gravity was derived and a new model generated in order to re-analyze the Fe I lines until convergence between model abundance and derived abundance. For these relatively well-studied red giants, no more than two iterations were required until convergence. Table 2 presents, for these red giants, the derived parameters $T_{\mathrm{eff}}, \log \left(L / L_{\odot}\right)$, mass, $\log g$, microturbulent velocity ( $\xi$; see Section 4.2 ), and overall metallicity, which is represented by $[\mathrm{Fe} / \mathrm{H}]$ as discussed in Section 4.2.

\subsection{Stellar Parameters and Evolutionary State}

Given the position of the stars in the $\log L$ versus $T_{\text {eff }}$ plane shown in Figure 2, it can be ascertained that four stars ( $\alpha$ Boo, $\mu$ Leo, $\delta$ Oph, and $\beta$ And) are most likely first-ascent red giants of low to moderate mass. Based on its warmer temperature and somewhat lower luminosity, it may be more likely that $\mu$ Leo is in or very near the core-He burning phase (clump giant) and has thus already ascended the RGB and experienced the core-He flash. It is also possible that the other three stars already noted above may be post-He core-burning stars ascending the AGB; however, lifetimes on the two separate evolutionary sequences would favor them as being on the RGB. All fall above the "luminosity bump" (Fusi Pecci et al. 1990) and would have thus experienced any extra mixing that may occur during that phase of stellar evolution. 


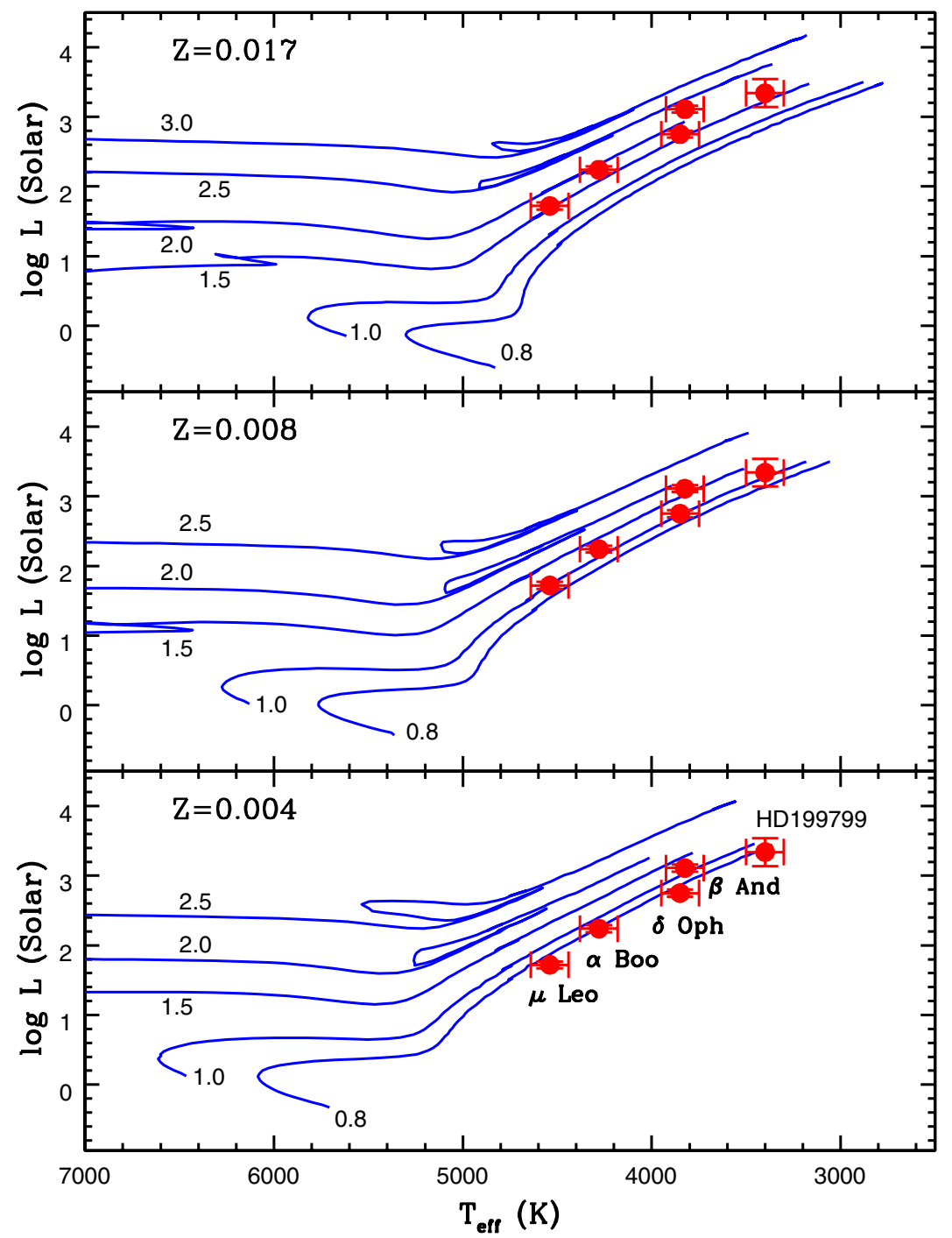

Figure 2. Positions of the field red giants in the $\log \left(L / L_{\odot}\right)-T_{\text {eff }}$ plane, which is a modified H-R diagram. The continuous curves represent stellar evolution tracks for different mass stars with each panel representing a different heavy-element mass fraction $(Z)$; this is equivalent to overall stellar metallicity with the Sun having $Z=$ 0.0145 (Lodders 2010). The model tracks are used to set $\log g$ for each red giant studied here.

(A color version of this figure is available in the online journal.)

Table 2

Red Giant Standard Stars: Derived Parameters

\begin{tabular}{lcccccc}
\hline \hline Star & $\begin{array}{c}\log \\
\left(L / L_{\odot}\right)\end{array}$ & $M / M_{\odot}$ & $\begin{array}{c}T_{\text {eff }} \\
(\mathrm{K})\end{array}$ & $\begin{array}{c}\log g \\
\left(\mathrm{~cm} \mathrm{~s}^{-2}\right)\end{array}$ & $\begin{array}{c}\xi \\
\left(\mathrm{km} \mathrm{s}^{-1}\right)\end{array}$ & $\begin{array}{c}{[\mathrm{Fe} / \mathrm{H}]^{\mathrm{a}}} \\
\beta \text { And }\end{array}$ \\
\hline Leo & $3.11 \pm 0.05$ & $1.9 \pm 0.2$ & $3825 \pm 75$ & $0.9 \pm 0.1$ & $2.20 \pm 0.10$ & -0.2 \\
$\alpha$ Boo & $2.68 \pm 0.03$ & $2.0 \pm 0.3$ & $4550 \pm 50$ & $2.1 \pm 0.1$ & $1.80 \pm 0.15$ & +0.3 \\
$\delta$ Oph & $2.24 \pm 0.02$ & $1.1 \pm 0.2$ & $4275 \pm 50$ & $1.7 \pm 0.1$ & $1.85 \pm 0.05$ & -0.4 \\
HD 199799 & $2.75 \pm 0.03$ & $1.5 \pm 0.2$ & $3850 \pm 75$ & $1.2 \pm 0.1$ & $1.90 \pm 0.10$ & +0.0 \\
\hline
\end{tabular}

Note. ${ }^{a}[\mathrm{Fe} / \mathrm{H}]$ represents the overall metallicity used in the final model atmosphere. Final Fe abundances are given in Table 6.

The coolest and most luminous red giant in this sample, HD199799, is an AGB star that is experiencing the third dredge-up, as evidenced by the observation of Tc I in its spectrum and its elevated $s$-process abundances, such as $[\mathrm{Y} / \mathrm{Fe}] \sim+0.5$ or $[\mathrm{Nd} / \mathrm{Fe}] \sim+0.4$ (Smith \& Lambert 1988 , 1990).

\section{THE ABUNDANCE ANALYSIS}

As with the initial abundances to be derived from APOGEE, the analysis here will rely on 1-D model atmospheres computed in LTE. Within APOGEE, the choice was made to base the initial abundance determinations on an extensive model atmosphere database generated using the ATLAS9 code (Kurucz 1993), thus the model atmospheres used here are based on these APOGEE models. ATLAS9 is widely used as a universal LTE 1D planeparallel atmosphere modeling code. The details of constructing the atmosphere database for APOGEE using ATLAS9 are presented in Meszaros et al. (2012).

The individual elemental abundances themselves will also be derived under the assumption of LTE with the spectrum 
Table 3

Fe I Lines used in the Abundance Determinations

\begin{tabular}{lccccccc}
\hline \hline $\begin{array}{l}\lambda \\
(\AA)\end{array}$ & $\begin{array}{c}\chi \\
(\mathrm{eV})\end{array}$ & $\log g f$ & $\alpha$ Boo & $\beta$ And & $\delta$ Oph & $\mu$ Leo & HD 199799 \\
\hline 15194.492 & 2.223 & -4.779 & 7.04 & 7.22 & 7.37 & 7.83 & 7.05 \\
15207.526 & 5.385 & +0.080 & 7.04 & 7.29 & 7.40 & $\mathrm{bl}^{\mathrm{a}}$ & 7.20 \\
15395.718 & 5.620 & -0.341 & 6.96 & 7.27 & $\mathrm{bl}^{\mathrm{a}}$ & 7.88 & 7.42 \\
15490.339 & 2.198 & -4.807 & 6.99 & 7.23 & 7.55 & 7.76 & 7.50 \\
15648.510 & 5.426 & -0.701 & 6.97 & 7.20 & 7.35 & 7.75 & 7.03 \\
15964.867 & 5.921 & -0.128 & 6.93 & 7.22 & 7.35 & 7.65 & 7.33 \\
16040.657 & 5.874 & +0.066 & 6.94 & 7.20 & $\mathrm{bl}$ & 7.69 & 7.14 \\
16153.247 & 5.351 & -0.743 & 6.94 & 7.22 & 7.53 & 7.75 & 7.32 \\
16165.032 & 6.319 & +0.723 & 6.98 & 7.24 & 7.56 & 7.73 & 7.30 \\
\hline
\end{tabular}

Note. ${ }^{\text {a }}$ bl: feature too blended to use in cooler or very metal-rich red giants.

synthesis code MOOG as the analysis tool (Sneden 1973 is the original reference, but updated information, data, and the most recent versions of the code can be found at http://www.as.utexas.edu/ chris/moog.html).

An extensive effort was made to produce as complete a spectral linelist as possible, so the analysis technique used here, as in APOGEE in general, is to utilize spectrum synthesis in a quantitative comparison between synthetic and observed spectra. All abundances are determined for each spectral line, or group of closely spaced molecular lines, via minimizing the residuals between observed and synthetic spectra as a function of the abundance in question.

\subsection{Choice of Fe I Lines and the Microturbulence}

Due to both its relatively large cosmic abundance and large number of energy levels, iron has historically been used as a diagnostic species for certain stellar parameters, as well as an overall metallicity indicator, so the results for Fe I lines selected from the APOGEE linelist (M. Shetrone et al., in preparation) are discussed here. In particular, Fe I lines are well suited for setting the microturbulent velocity, $\xi$, which is required for 1-D model atmosphere abundance analyses.

A large number of Fe I lines fall within the APOGEE spectral window (15100-16900 $\AA$ ), although there are no Fe II lines strong enough to be detected in these red giants. Since all abundance determinations are done via spectrum synthesis, the $\mathrm{Fe}$ I lines were culled to include those lines that contain only contributions from Fe I (this was determined by preliminary synthesis of the red giant spectra, then setting the Fe abundance to zero and ensuring that the spectral feature in question vanished); since the Fe I lines are so numerous, this stringent selection still results in an adequate list of lines. Table 3 presents the $\mathrm{Fe}$ I lines used for the iron abundance determinations. As is true of most of the atomic lines in the $H$ band, the excitation energies are dominated by rather high-excitation lines, although for Fe I there are two lower-excitation lines.

The Fe I lines span a large enough range in line strength that they can be used to set the microturbulent velocity. This parameter is defined by the value of $\xi$ that produces no trend in the Fe abundance as a function of line strength. For a range of microturbulent velocities Fe abundances were determined from each $\mathrm{Fe}_{\mathrm{I}}$ line and the adopted value of $\xi$ was one in which a linear regression between $A(\mathrm{Fe})$ as a function of $\log \left(W_{\lambda} / \lambda\right)$ yielded a slope of zero. This procedure is illustrated graphically in Figure 3, where the abundances derived for each individual line are plotted versus $\xi$ (the continuous lines); the circle with

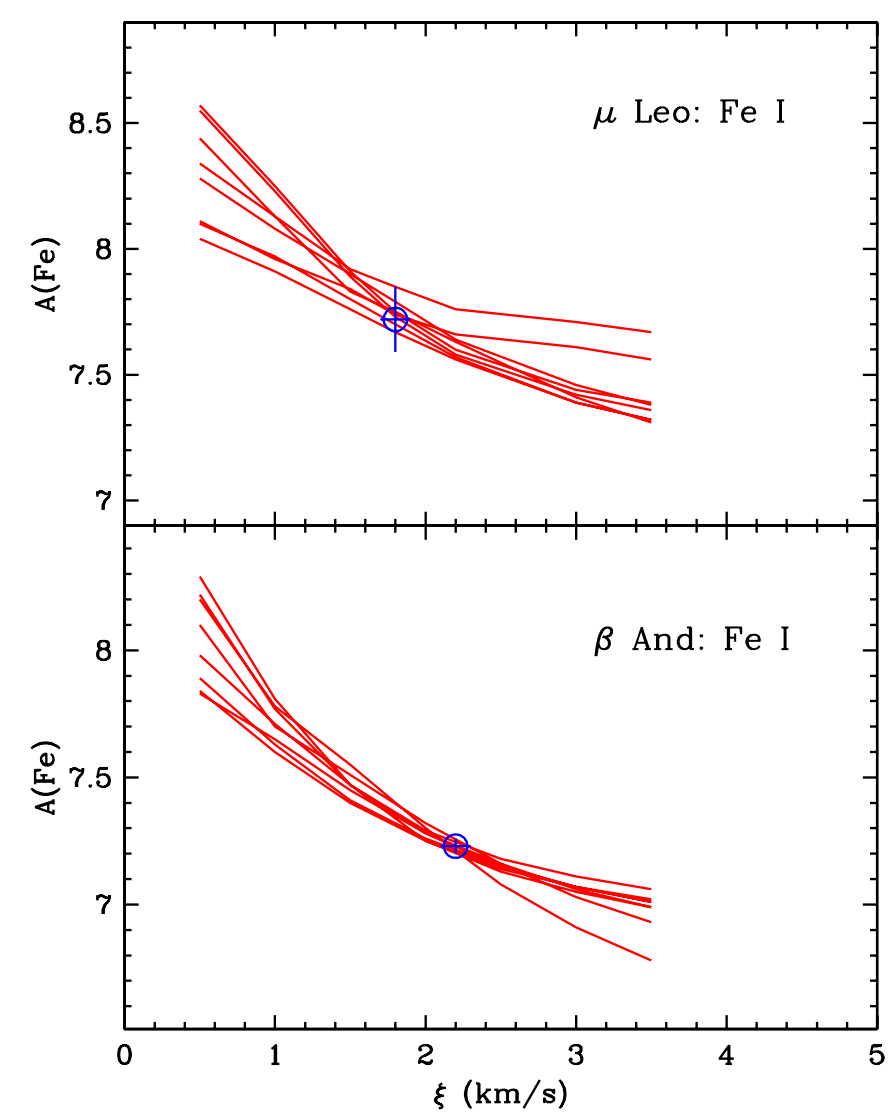

Figure 3. Individual Fe I abundances as a function of microturbulent velocity for $\mu$ Leo (top panel) and $\beta$ And (bottom panel). Each curve represents a single $\mathrm{Fe}_{\mathrm{I}}$ line. Stronger lines are more sensitive to changes in $\xi$ than weaker lines. The value of $\xi$ that yields no significant abundance differences as a function of reduced line strength $\left(\log W_{\lambda} / \lambda\right)$ is taken as the characteristic microturbulent velocity for the star. The derived microturbulent velocity as displayed here also corresponds to the smallest scatter in $A(\mathrm{Fe})$.

(A color version of this figure is available in the online journal.)

error bars shows where the slope of $A(\mathrm{Fe})$ with line strength goes to zero.

The microturbulent velocities set by the Fe I lines, as well as the Fe abundances are listed in Table 2 as part of the derived set of stellar parameters. The individual line-by-line abundances as determined for each Fe I line are also listed in Table 3. The Fe abundances were used to set the final overall metallicities of the ATLAS9 model atmospheres for the analysis of additional elements via atomic or molecular lines.

\subsection{The $C, N$, and $O$ Abundances}

Abundances for ${ }^{12} \mathrm{C},{ }^{14} \mathrm{~N},{ }^{16} \mathrm{O}$, and the minor carbon isotope ${ }^{13} \mathrm{C}$ are derived from combinations of vibration-rotation $(\mathrm{V}-\mathrm{R})$ lines of $\mathrm{CO}\left(\mathrm{X}^{1} \Sigma^{+}\right)$and $\mathrm{OH}\left(\mathrm{X}^{2} \Pi\right)$, along with electronic transitions of $\mathrm{CN}\left(\mathrm{A}^{2} \Pi-\mathrm{X}^{2} \Sigma\right)$. Although the details of the ingredients that went into these molecular lines in the APOGEE linelist will be found in M. Shetrone et al. (in preparation), a few highlights are noted here. The adopted dissociation energies $\left(\mathrm{D}_{0}\right)$ are $11.092 \mathrm{eV}$ for $\mathrm{CO}, 4.395 \mathrm{eV}$ for $\mathrm{OH}$, and $7.76 \mathrm{eV}$ for $\mathrm{CN}$. The $g f$-values are from Goorvitch (1994) for CO, Goldman et al. (1998) for $\mathrm{OH}$, and the $\mathrm{CN} g f$-values are taken from the baseline linelist of Kurucz (1993), with updates to these values from the prescription given in Melendez \& Barbuy (1999).

The procedure used for the $\mathrm{CNO}$ analysis, since all red giants studied here have $\mathrm{C} / \mathrm{O} \leqslant 1$, is to use $\mathrm{CO}$ to set the carbon 
Table 4

Molecular Lines and Features used for ${ }^{12} \mathrm{C},{ }^{13} \mathrm{C},{ }^{14} \mathrm{~N}$, and ${ }^{16} \mathrm{O}$

\begin{tabular}{|c|c|c|c|c|c|c|}
\hline Molecular Lines & $\begin{array}{c}\lambda \text {-interval } \\
(\AA)\end{array}$ & $\alpha$ Boo & $\beta$ And & $\delta \mathrm{Oph}$ & $\mu$ Leo & HD 199799 \\
\hline \multicolumn{7}{|c|}{${ }^{12} \mathrm{C}$ from ${ }^{12} \mathrm{C}^{16} \mathrm{O}$ lines } \\
\hline$(3-0) \mathrm{V}-\mathrm{R}$ & $15578-15586$ & 7.86 & 8.05 & 8.27 & $\mathrm{w}^{\mathrm{a}}$ & 8.51 \\
\hline$(4-1) \mathrm{V}-\mathrm{R}$ & 15774-15787 & 7.97 & 8.11 & 8.24 & 8.46 & 8.51 \\
\hline$(5-2) \mathrm{V}-\mathrm{R}$ & 15976-16000 & 7.90 & 8.07 & 8.23 & 8.56 & 8.38 \\
\hline$(6-3) \mathrm{V}-\mathrm{R}$ & $16183-16196$ & 7.90 & 8.03 & 8.22 & 8.54 & 8.43 \\
\hline \multicolumn{7}{|c|}{${ }^{12} \mathrm{C} /{ }^{13} \mathrm{C}$ ratios from ${ }^{13} \mathrm{C}^{16} \mathrm{O}$ and ${ }^{13} \mathrm{C}^{14} \mathrm{~N}$ lines } \\
\hline${ }^{13} \mathrm{C}^{16} \mathrm{O}(3-0) \mathrm{V}-\mathrm{R}$ & $15922-15926$ & 7 & 17 & 14 & $\mathrm{w}^{\mathrm{a}}$ & 30 \\
\hline${ }^{13} \mathrm{C}^{16} \mathrm{O}(4-1) \mathrm{V}-\mathrm{R}$ & $16120-16125$ & 6 & 15 & 11 & $w^{a}$ & 32 \\
\hline${ }^{13} \mathrm{C}^{16} \mathrm{O}(6-3) \mathrm{V}-\mathrm{R}$ & $16740-16747$ & 6 & 13 & 11 & $\mathrm{w}^{\mathrm{a}}$ & 20 \\
\hline${ }^{13} \mathrm{C}^{14} \mathrm{~N}$ & $15314-15315$ & $\mathrm{w}^{\mathrm{a}}$ & $\mathrm{w}^{\mathrm{a}}$ & $\mathrm{w}^{\mathrm{a}}$ & 22 & $w^{a}$ \\
\hline${ }^{13} \mathrm{C}^{14} \mathrm{~N}$ & $15354-15356$ & $\mathrm{w}^{\mathrm{a}}$ & $\mathrm{w}^{\mathrm{a}}$ & $\mathrm{w}^{\mathrm{a}}$ & 17 & $w^{\mathrm{a}}$ \\
\hline \multicolumn{7}{|c|}{${ }^{16} \mathrm{O}$ from ${ }^{16} \mathrm{OH}$ lines } \\
\hline$(2-0) \mathrm{P}_{1} 9.5$ & $15277-15282$ & 8.55 & 8.83 & 8.78 & 9.10 & 8.80 \\
\hline$(3-1) \mathrm{P}_{2} 3.5$ & 15390-15392 & 8.54 & 8.78 & 8.76 & 8.93 & 8.60 \\
\hline$(3-1) \mathrm{P}_{2} 4.5$ & 15504-15507 & 8.48 & 8.71 & 8.73 & 9.15 & 8.58 \\
\hline$(2-0) \mathrm{P}_{2} 11.5$ & $15568-15573$ & 8.51 & 8.80 & 8.78 & 8.98 & 8.52 \\
\hline$(3-1) \mathrm{P}_{2} 9.5$ & $16189-16193$ & 8.47 & 8.78 & 8.81 & 9.07 & 8.63 \\
\hline \multicolumn{7}{|c|}{${ }^{14} \mathrm{~N}$ from ${ }^{12} \mathrm{C}^{14} \mathrm{~N}$ lines } \\
\hline$(1-2)$ Q2 41.5 & 15260. & 7.61 & 8.10 & 8.23 & 8.83 & 8.31 \\
\hline (1-2) P2 34.5 & 15322. & 7.68 & 8.07 & 8.21 & 8.88 & 8.35 \\
\hline (1-2) R2 56.5 & 15397. & 7.65 & 8.06 & 8.26 & 8.63 & 8.28 \\
\hline$(0-1)$ R1 68.5 & 15332. & 7.63 & 7.95 & $\mathrm{bl}^{\mathrm{b}}$ & 8.68 & 8.35 \\
\hline$(0-1)$ P2 49.5 & 15410. & 7.66 & 8.03 & 8.13 & 8.80 & $\mathrm{bl}^{\mathrm{b}}$ \\
\hline$(0-1)$ Q2 59.5 & 15447. & 7.64 & 7.98 & 8.28 & 8.73 & $\mathrm{bl}^{\mathrm{b}}$ \\
\hline$(0-1)$ Q1 60.5 & 15466. & 7.65 & 8.06 & 8.23 & 8.51 & $\mathrm{bl}^{\mathrm{b}}$ \\
\hline (1-2) P2 38.5 & 15472. & 7.71 & 8.13 & 8.11 & 8.68 & 8.25 \\
\hline (0-1) P1 51.5 & 15482. & 7.56 & 8.03 & 8.15 & 8.68 & 8.25 \\
\hline
\end{tabular}

Notes.

${ }^{\mathrm{a}} \mathrm{w}$ : feature too weak to use.

${ }^{\mathrm{b}}$ bl: feature too blended by nearby lines.

abundance. With that carbon abundance, $\mathrm{OH}$ provides an $\mathrm{O}$ abundance, which, if different from the initial value (taken to be the solar value scaled by the stellar value of $[\mathrm{Fe} / \mathrm{H}])$, is used to re-analyze the $\mathrm{CO}$ lines. This process is repeated until the $\mathrm{C}$ and $\mathrm{O}$ abundances yield consistent abundances from $\mathrm{CO}$ and $\mathrm{OH}$. With these values of $\mathrm{C}$ and $\mathrm{O}$, the $\mathrm{CN}$ lines are used to derive the abundance of nitrogen. In general, the abundance of $\mathrm{N}$ has little to no effect on the $\mathrm{CO}$ and $\mathrm{OH}$ lines, however, the respective final $\mathrm{C}, \mathrm{N}$, and $\mathrm{O}$ abundances all provide self-consistent results from $\mathrm{CO}, \mathrm{OH}$, and $\mathrm{CN}$.

All spectral features were synthesized and the molecular regions that were used to extract the abundances are listed in Table 4. In this case, if a range of wavelengths is given it indicates that a spectral window containing numerous molecular lines was used (for $\mathrm{CO}$ and $\mathrm{OH}$ ), while for $\mathrm{CN}$ individual transitions were identified and synthesized. As with Table 3, the abundances derived for each feature are also listed in Table 4. In the case of ${ }^{13} \mathrm{C}$, as has been customary for minor isotopes, the abundance is listed as the ratio of ${ }^{12} \mathrm{C} /{ }^{13} \mathrm{C}$, with $A\left({ }^{12} \mathrm{C}\right)$ being set by the mean abundance of carbon-12.

\subsection{The Other Atomic Lines and Elemental Abundances}

With the $\mathrm{Fe}$ and $\mathrm{C}, \mathrm{N}$, and $\mathrm{O}$ abundances in hand, additional elements are derived via atomic spectral lines in the wavelength interval 15100-16900 ̊. The APOGEE linelist was searched for suitable species, all neutral over the $T_{\text {eff }}$ range of the red giants here, and some 12 other elements were deemed detectable and able to be analyzed quantitatively: $\mathrm{Mg}$ I, Al I, Si I, KI, Ca I, Ti I, Vi, Cri, Mni, Co I, Ni I, and CuI. The additional atomic lines are listed in Table 5. As with the molecular lines, if a precise wavelength is shown, the transition in question is represented by a single line, however, if the wavelength is approximated by an integer value it indicates multiple transitions due to hyperfine (hfs) splitting or isotopic splitting. In the case of isotopic components, solar-system isotopic fractions are assumed and, in general, this assumption has no significant effect on the derived total atomic abundances. Figure 4 illustrates the fitting procedure for an atomic line from $\mathrm{Mn} \mathrm{I}$ in $\delta \mathrm{Oph}$, with the underlying hfs components illustrated, as well as nearby blending features, with several synthetic spectra plotted which have differing Mn abundances. As with the previous Tables 3 and 4, individual abundances are provided for each line or feature. As noted previously, the details of the generation of the APOGEE linelist will be found in M. Shetrone et al. (in preparation).

Table 6 provides a summary table of all abundances listed as their mean values and standard deviations from the lines or spectral intervals shown in Tables 3- 5. In cases where only one line or region was observed, no standard deviation is given. The abundance of ${ }^{13} \mathrm{C}$ is given as the isotopic ratio of ${ }^{12} \mathrm{C} /{ }^{13} \mathrm{C}$. 
Table 5

Atomic Lines used and Derived Abundance

\begin{tabular}{|c|c|c|c|c|c|c|c|c|}
\hline Element & $\begin{array}{c}\lambda \\
(\AA)\end{array}$ & $\begin{array}{c}\chi \\
(\mathrm{eV})\end{array}$ & $\log g f$ & $\alpha$ Boo & $\beta$ And & $\delta \mathrm{Oph}$ & $\mu$ Leo & HD 199799 \\
\hline \multirow[t]{7}{*}{$\overline{\mathrm{Mg}_{\mathrm{I}}}$} & 15740.716 & 5.931 & -0.262 & 7.15 & 7.25 & 7.53 & 7.80 & 7.29 \\
\hline & 15748.9 & 5.932 & +0.276 & 7.09 & $\mathrm{~s}^{\mathrm{a}}$ & 7.57 & $s^{a}$ & 7.23 \\
\hline & 15765.8 & 5.933 & +0.504 & 7.04 & $s^{a}$ & $\mathrm{~s}^{\mathrm{a}}$ & 7.87 & $\mathrm{bl}^{\mathrm{b}}$ \\
\hline & 15879.5 & 5.946 & -1.248 & 7.13 & 7.20 & 7.45 & 7.82 & $\mathrm{bl}^{\mathrm{b}}$ \\
\hline & 15886.2 & 5.946 & -1.555 & 7.22 & 7.21 & 7.53 & $\mathrm{bl}^{\mathrm{b}}$ & 7.18 \\
\hline & 15889.485 & 5.946 & -2.013 & $\mathrm{bl}^{\mathrm{b}}$ & 7.30 & 7.55 & 7.90 & $\mathrm{w}^{\mathrm{c}}$ \\
\hline & 15954.477 & 6.588 & -0.807 & 7.25 & 7.36 & 7.61 & $\mathrm{bl}^{\mathrm{b}}$ & 7.45 \\
\hline \multirow[t]{2}{*}{ Al I } & 16718.957 & 4.085 & +0.290 & 6.15 & 6.15 & $\mathrm{~s}^{\mathrm{a}}$ & 6.85 & $\mathrm{bl}^{\mathrm{b}}$ \\
\hline & 16763.359 & 4.087 & -0.524 & 6.16 & 6.09 & 6.45 & 6.94 & 6.10 \\
\hline \multirow[t]{9}{*}{ Si I } & 15361.161 & 5.954 & -1.925 & 6.99 & $\mathrm{bl}^{\mathrm{b}}$ & $\mathrm{bl}^{\mathrm{b}}$ & $\mathrm{bl}^{\mathrm{b}}$ & $w^{c}$ \\
\hline & 15376.831 & 6.222 & -0.649 & 7.18 & 7.17 & 7.57 & 7.66 & $\mathrm{w}^{\mathrm{c}}$ \\
\hline & 15833.602 & 6.222 & -0.168 & 6.93 & 6.95 & 7.28 & 7.72 & 7.20 \\
\hline & 15960.063 & 5.984 & +0.107 & 7.07 & 7.14 & 7.43 & 7.88 & $\mathrm{w}^{\mathrm{c}}$ \\
\hline & 16060.009 & 5.954 & -0.566 & 7.21 & 7.25 & 7.59 & 7.74 & 7.33 \\
\hline & 16094.787 & 5.964 & -0.168 & 7.11 & 7.06 & 7.46 & 7.66 & 7.32 \\
\hline & 16215.670 & 5.964 & -0.665 & 7.15 & 7.23 & 7.62 & $\mathrm{bl}^{\mathrm{b}}$ & $\mathrm{bl}^{\mathrm{b}}$ \\
\hline & 16680.770 & 5.984 & -0.140 & 7.11 & 7.25 & 7.62 & 7.83 & 7.35 \\
\hline & 16828.159 & 5.984 & -1.102 & 7.13 & 7.17 & 7.45 & 7.85 & $\mathrm{bl}^{\mathrm{b}}$ \\
\hline \multirow[t]{2}{*}{$\mathrm{K}_{\mathrm{I}}$} & 15163.067 & 2.670 & +0.524 & 4.81 & 4.82 & 5.18 & 5.63 & 5.20 \\
\hline & 15168.376 & 2.670 & +0.347 & 4.77 & 4.89 & $\mathrm{bl}^{\mathrm{b}}$ & $\mathrm{bl}^{\mathrm{b}}$ & $\mathrm{bl}^{\mathrm{b}}$ \\
\hline \multirow[t]{4}{*}{$\mathrm{Ca} \mathrm{I}$} & 16136.823 & 4.531 & -0.552 & 5.75 & 6.09 & 6.23 & 6.69 & 6.06 \\
\hline & 16150.763 & 4.532 & -0.229 & 5.88 & 5.95 & 6.21 & 6.53 & 6.07 \\
\hline & 16155.236 & 4.532 & -0.619 & 5.82 & 6.02 & 6.30 & 6.61 & 6.16 \\
\hline & 16157.364 & 4.554 & -0.208 & 5.90 & 6.02 & 6.23 & 6.63 & 6.16 \\
\hline \multirow[t]{5}{*}{ Ti I } & 15543.756 & 1.879 & -1.120 & 4.53 & 4.74 & 5.05 & 5.58 & 4.74 \\
\hline & 15602.842 & 2.267 & -1.643 & 4.66 & 4.74 & 5.03 & 5.43 & $\mathrm{bl}^{\mathrm{b}}$ \\
\hline & 15698.979 & 1.887 & -2.060 & 4.52 & 4.69 & 5.03 & 5.13 & $\mathrm{bl}^{\mathrm{b}}$ \\
\hline & 15715.573 & 1.873 & -1.250 & 4.57 & 4.71 & 5.14 & $\mathrm{bl}^{\mathrm{b}}$ & 4.70 \\
\hline & 16635.161 & 2.345 & -1.807 & 4.65 & $\mathrm{bl}^{\mathrm{b}}$ & 5.10 & 5.46 & 4.90 \\
\hline $\mathrm{V}_{\mathrm{I}}$ & 15924. & 2.138 & -1.175 & 3.61 & 3.66 & 3.86 & 4.18 & $\mathrm{bl}^{\mathrm{b}}$ \\
\hline \multirow[t]{2}{*}{ Cr I } & 15680.063 & 4.697 & +0.179 & 5.08 & 5.38 & 5.62 & 6.11 & 5.20 \\
\hline & 15860.214 & 4.697 & -0.012 & 5.14 & 5.39 & 5.57 & 6.16 & 5.54 \\
\hline \multirow[t]{3}{*}{ Mn I } & 15159. & 4.889 & +0.544 & 4.88 & 5.19 & $\mathrm{bl}^{\mathrm{b}}$ & 5.82 & $\mathrm{bl}^{\mathrm{b}}$ \\
\hline & 15217. & 4.889 & +0.414 & 4.91 & 5.26 & 5.47 & 5.83 & 5.32 \\
\hline & 15262. & 4.889 & +0.345 & 4.78 & 5.08 & 5.21 & 5.71 & 5.30 \\
\hline CoI & 16757.7 & 3.409 & -1.230 & 4.50 & 4.73 & 4.85 & 5.23 & 4.50 \\
\hline \multirow[t]{7}{*}{$\mathrm{Ni}$ I } & 15605.680 & 5.299 & -0.376 & 5.76 & 6.08 & 6.18 & 6.69 & 6.05 \\
\hline & 15632.654 & 5.305 & -0.106 & 5.82 & 5.99 & 6.20 & 6.65 & 6.02 \\
\hline & 16584.439 & 5.299 & -0.528 & 5.71 & 6.00 & 6.21 & 6.58 & 6.12 \\
\hline & 16589.295 & 5.469 & -0.600 & 5.81 & 6.03 & 6.23 & 6.72 & 5.98 \\
\hline & 16673.711 & 6.029 & +0.317 & 5.72 & $\mathrm{bl}^{\mathrm{b}}$ & $\mathrm{bl}^{\mathrm{b}}$ & 6.39 & 6.12 \\
\hline & 16815.471 & 5.305 & -0.606 & 5.79 & 5.94 & 6.06 & 6.58 & 5.95 \\
\hline & 16818.760 & 6.039 & +0.311 & 5.77 & $\mathrm{bl}^{\mathrm{b}}$ & 6.22 & $\mathrm{bl}^{\mathrm{b}}$ & 5.95 \\
\hline $\mathrm{Cu} \mathrm{I}$ & 16005.7 & 5.348 & -0.131 & 3.55 & 4.01 & 4.18 & 4.41 & 4.20 \\
\hline
\end{tabular}

Notes.

${ }^{\mathrm{a}} \mathrm{s}$ : feature too strong to use.

${ }^{\mathrm{b}}$ bl: feature too blended by nearby lines.

${ }^{c}$ w: feature too weak.

\subsection{Abundance Sensitivity to Stellar Parameters}

Most of the abundances presented here are derived from more than one atomic or molecular line or features of lines, thus internal consistency in the average abundances is defined by the scatter from the individual features. This scatter is characterized by the standard deviations of Table 6 . Because multiple features arise from differing line strengths (weak versus strong), excitation potentials, or ionization fractions, these respective standard deviations provide some estimate as to how well the 1-D LTE analysis can recover the details of the underlying stellar spectrum from the basic model atmosphere parameters $T_{\text {eff }}, \log g$, metallicity, and derived value of $\xi$.
The typical standard deviations from Table 6 are $\sim \pm 0.05$ dex. Of note is the metal-rich K-giant $\mu$ Leo, which exhibits exceptionally strong $\mathrm{CN}$ lines, that are numerous throughout this $H$-band window. The background $\mathrm{CN}$ absorption creates blending which lowers the overall signature of other abundances and increases the uncertainty in these other abundances; this is reflected in the generally larger standard deviations found in the $\mu$ Leo abundances.

Besides internal consistency from the various spectral lines of different species, it is useful to explore their sensitivities to stellar parameters in order to characterize the magnitudes of possible systematic effects due to uncertainties in the four primary parameters of effective temperature, surface gravity, 


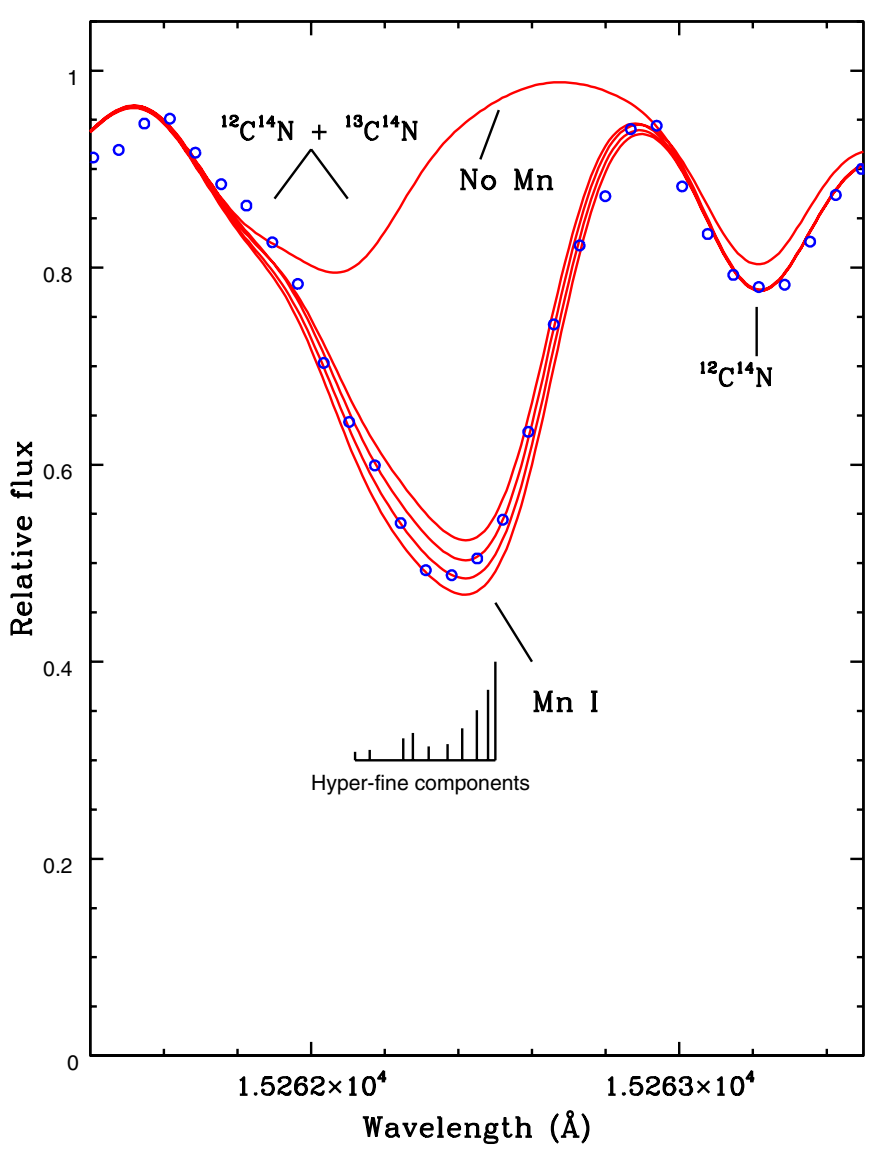

Figure 4. Comparison of the observed spectrum near the Mn I line at $15262 \AA$ in $\delta$ Oph (blue open circles) with synthetic spectra which were computed for Mn abundances separated in intervals of 0.1 dex. This Mn I transition consists of a number of hfs components of differing wavelengths, as shown, with the vertical length of each component proportional to the $g f$-value of that component transition. Note that $\mathrm{Mn} \mathrm{I}$ is blended with a $\mathrm{CN}$ feature containing both ${ }^{12} \mathrm{C}^{14} \mathrm{~N}$ and ${ }^{13} \mathrm{C}^{14} \mathrm{~N}$ lines, thus the value of ${ }^{12} \mathrm{C} /{ }^{13} \mathrm{C}$ ratio must be included. Both the hfs structure and $\mathrm{CN}$ blending illustrate the need for the APOGEE analysis to be based on spectrum synthesis with an accurate linelist.

(A color version of this figure is available in the online journal.)

metallicity, and 1-D derived microturbulent velocity. For this discussion $T_{\text {eff }}$ is written as $T, \log g$ as $\mathrm{G}$, model atmosphere metallicity $([\mathrm{m} / \mathrm{H}])$ as $\mathrm{m}$, and microturbulent velocity as $\xi$. Because the abundance for a given element, $A=\log [N(\mathrm{~A}) / N(\mathrm{H})]$ $+12 .=A(\mathrm{~T}, \mathrm{G}, \mathrm{m}, \xi)$, the incremental change in this abundance due to small variations in the parameters is given by

$d A=(\partial A / \partial T) d T+(\partial A / \partial G) d G+(\partial A / \partial \xi) d \xi+(\partial A / \partial m) d m$.

The scatter in the abundance caused by small changes in the stellar parameters is then defined as

$$
\sigma_{\mathrm{A}}^{2}=\left\langle d A^{2}\right\rangle
$$

and $d A^{2}$ can be written as

$$
\begin{aligned}
(d A)^{2}= & {[(\partial A / \partial T) d T]^{2}+[(\partial A / \partial G) d G]^{2} } \\
& +[(\partial A / \partial \xi) d \xi]^{2}+[(\partial A / \partial m) d m]^{2} \\
& +(\partial A / \partial T)(\partial A / \partial G)[d T d G+d G d T] \\
& +(\partial A / \partial T)(\partial A / \partial \xi)[d T d \xi+d \xi d T] \\
& +(\partial A / \partial T)(\partial A / \partial m)[d T d m+d m d T] \\
& +(\partial A / \partial G)(\partial A / \partial \xi)[d G d \xi+d \xi d G] \\
& +(\partial A / \partial G)(\partial A / \partial m)[d G d m+d m d G] \\
& +(\partial A / \partial \xi)(\partial A / \partial m)[d \xi d m+d m d \xi] .
\end{aligned}
$$

Table 6

Chemical Abundances

\begin{tabular}{lccccc}
\hline \hline Element & $\alpha$ Boo & $\beta$ And & $\delta$ Oph & $\mu$ Leo & HD 199799 \\
\hline $\mathrm{Fe}$ & $6.98 \pm 0.04$ & $7.23 \pm 0.03$ & $7.44 \pm 0.09$ & $7.76 \pm 0.08$ & $7.25 \pm 0.12$ \\
${ }^{12} \mathrm{C}$ & $7.96 \pm 0.05$ & $8.06 \pm 0.03$ & $8.24 \pm 0.02$ & $8.52 \pm 0.05$ & $8.46 \pm 0.06$ \\
${ }^{12} \mathrm{C} /{ }^{13} \mathrm{C}$ & $6.3 \pm 0.6$ & $15 \pm 2$ & $12 \pm 2$ & $20 \pm 3$ & $27 \pm 6$ \\
${ }^{14} \mathrm{~N}$ & $7.64 \pm 0.04$ & $8.05 \pm 0.06$ & $8.20 \pm 0.06$ & $8.71 \pm 0.11$ & $8.31 \pm 0.05$ \\
${ }^{16} \mathrm{O}$ & $8.64 \pm 0.04$ & $8.78 \pm 0.04$ & $8.77 \pm 0.03$ & $9.05 \pm 0.09$ & $8.63 \pm 0.09$ \\
$\mathrm{Mg}$ & $7.15 \pm 0.08$ & $7.26 \pm 0.07$ & $7.54 \pm 0.05$ & $7.85 \pm 0.05$ & $7.28 \pm 0.11$ \\
$\mathrm{Al}$ & $6.16 \pm 0.01$ & $6.12 \pm 0.03$ & 6.45 & $6.90 \pm 0.05$ & 6.10 \\
$\mathrm{Si}$ & $7.12 \pm 0.07$ & $7.18 \pm 0.07$ & $7.53 \pm 0.08$ & $7.76 \pm 0.09$ & $7.30 \pm 0.07$ \\
$\mathrm{~K}$ & $4.79 \pm 0.02$ & $4.86 \pm 0.04$ & 5.18 & 5.63 & 5.20 \\
$\mathrm{Ca}$ & $5.84 \pm 0.07$ & $6.02 \pm 0.06$ & $6.24 \pm 0.04$ & $6.62 \pm 0.07$ & $6.11 \pm 0.05$ \\
$\mathrm{Ti}$ & $4.59 \pm 0.07$ & $4.72 \pm 0.02$ & $5.07 \pm 0.05$ & $5.40 \pm 0.19$ & $4.78 \pm 0.10$ \\
$\mathrm{~V}$ & 3.61 & 3.66 & 3.86 & 4.18 & $\ldots$ \\
$\mathrm{Cr}$ & $5.11 \pm 0.03$ & $5.38 \pm 0.01$ & $5.60 \pm 0.03$ & $6.14 \pm 0.03$ & $5.37 \pm 0.17$ \\
$\mathrm{Mn}$ & $4.86 \pm 0.07$ & $5.18 \pm 0.09$ & $5.34 \pm 0.13$ & $5.79 \pm 0.07$ & $5.31 \pm 0.01$ \\
$\mathrm{Co}$ & 4.50 & 4.73 & 4.85 & 5.23 & 4.50 \\
$\mathrm{Ni}$ & $5.77 \pm 0.04$ & $6.01 \pm 0.05$ & $6.18 \pm 0.06$ & $6.60 \pm 0.12$ & $6.03 \pm 0.07$ \\
$\mathrm{Cu}$ & 3.55 & 4.01 & 4.18 & 4.41 & 4.20 \\
\hline
\end{tabular}

The partial derivative terms are calculated by changing each model parameter and determining the resulting change in logarithmic abundance, $A$. Since the perturbation in stellar parameters is relatively small, the changes are approximated by linear trends. As this paper is a limited study of a few stars as a test of the APOGEE linelist, a test of abundance sensitivity to stellar parameters is carried out for a representative model taken with $T_{\text {eff }}=4000 \mathrm{~K}, \log g=1.3,[\mathrm{~m} / \mathrm{H}]=+0.0$, with $\xi=$ $2.0 \mathrm{~km} \mathrm{~s}^{-1}$. The predicted equivalent widths were generated for the lines used in the abundance analysis and the stellar parameters were then perturbed by $d T=+50 \mathrm{~K}, d G=+0.2 \mathrm{dex}$, $d m=+0.1 \mathrm{dex}$, and $d \xi=+0.2 \mathrm{~km} \mathrm{~s}^{-1}$, with new abundances derived for each separate perturbation, giving the change in abundance for each parameter change. These coefficients, which reflect the change in abundance, are listed in Table 7 and provide an overall view of how sensitive the derived abundances are to changes in stellar parameters that are representative of realistic errors for these particular red giants.

Columns 6 and 7 in Table 7 provide the values of $d A$ two cases: one using only the first four terms which contains the change in abundance due to changes in each primary parameter $\left(d A^{\prime}\right.$ in Column 6 , which is valid if all parameters are independent of each other), while Column 7 includes the covariant terms. Within the analysis technique applied here, it was found that the microturbulent velocity did not measurably depend on the derived effective temperature or gravity, and the $d T-d \xi$ and $d G-d \xi$ cross terms are not included. As can be seen by the comparison of $d A^{\prime}$ and $d A$, the covariant terms contribute significantly, in many species, to the uncertainty. The largest covariant terms are typically those between $T_{\text {eff }}$ and $\log g$, caused by the slope of the RGB in the $T_{\text {eff }}-\log \left(L / L_{\odot}\right)$ plane (since $L \sim T_{\text {eff }}^{4} / \mathrm{g}$ ).

\subsection{Comparison to Previously Published Abundances}

The abundances derived here rely on the APOGEE $H$-band spectral window from 15100 to $16900 \AA$, along with parallaxes and photometry. Historically, most detailed abundance patterns obtained from high-resolution spectroscopy have tended to be based on wavelengths below $10000 \AA$, so it is useful to compare the abundances based on the APOGEE linelist with those from other studies for stars in common. Within the sample of red giants discussed here, previous high-resolution abundance 
Table 7

Abundance Sensitivity: $T_{\text {eff }}=4000 \mathrm{~K}, \log g=1.3, \xi=2.0 \mathrm{~km} \mathrm{~s}^{-1},[\mathrm{~m} / \mathrm{H}]=0.0$

\begin{tabular}{|c|c|c|c|c|c|c|}
\hline Species & $\begin{array}{l}\partial A / \partial T \\
(+50 \mathrm{~K})\end{array}$ & $\begin{array}{c}\partial A / \partial G \\
(+0.2 \text { dex })\end{array}$ & $\begin{array}{c}\partial A / \partial \xi \\
\left(+0.2 \mathrm{~km} \mathrm{~s}^{-1}\right)\end{array}$ & $\begin{array}{c}\partial A / \partial m \\
(+0.1 \mathrm{dex})\end{array}$ & $d A^{\prime}$ & $d A$ \\
\hline $\mathrm{Fe} I$ & +0.015 & +0.003 & -0.056 & +0.032 & 0.066 & 0.087 \\
\hline $\mathrm{CO}$ & +0.032 & +0.072 & -0.011 & +0.041 & 0.089 & 0.167 \\
\hline $\mathrm{CN}$ & -0.018 & +0.064 & -0.006 & +0.038 & 0.077 & 0.127 \\
\hline $\mathrm{OH}$ & +0.124 & -0.022 & -0.079 & +0.111 & 0.186 & 0.363 \\
\hline $\mathrm{Mg}_{\mathrm{I}}$ & +0.017 & -0.031 & -0.042 & +0.029 & 0.062 & 0.098 \\
\hline Al I & +0.055 & -0.077 & -0.052 & +0.027 & 0.111 & 0.207 \\
\hline Si I & +0.003 & -0.013 & -0.039 & +0.038 & 0.056 & 0.065 \\
\hline $\mathrm{K}_{\mathrm{I}}$ & +0.042 & -0.046 & -0.048 & +0.014 & 0.081 & 0.143 \\
\hline $\mathrm{Ca} \mathrm{I}$ & +0.045 & -0.046 & -0.032 & +0.017 & 0.074 & 0.143 \\
\hline Ti I & +0.082 & -0.004 & -0.111 & +0.015 & 0.139 & 0.168 \\
\hline$V_{\text {I }}$ & +0.076 & +0.009 & -0.054 & +0.011 & 0.094 & 0.132 \\
\hline Cr I & +0.038 & -0.018 & -0.031 & +0.016 & 0.055 & 0.100 \\
\hline Mn I & +0.032 & -0.057 & -0.056 & +0.041 & 0.095 & 0.163 \\
\hline CoI & +0.026 & +0.033 & -0.075 & +0.034 & 0.092 & 0.136 \\
\hline $\mathrm{Ni}$ I & -0.005 & +0.017 & -0.043 & +0.031 & 0.056 & 0.068 \\
\hline $\mathrm{Cu} \mathrm{I}$ & +0.001 & +0.032 & -0.013 & +0.019 & 0.039 & 0.044 \\
\hline
\end{tabular}

analyses have been conducted on $\alpha$ Boo, $\mu$ Leo, $\beta$ And, and HD 199799.

\subsection{1. $\alpha$ Boo}

Being the nearest red giant, $\alpha$ Boo is often used as the standard star of choice for abundance comparisons. Ramirez \& Allende Prieto (2011) have produced a recent and detailed highresolution optical spectral analysis, reviewing both fundamental stellar parameters for this star, as well as deriving an extensive set of elemental abundances. They used the observed spectral energy distribution and compared to theoretical ones from the Kurucz grid of no-overshoot model atmospheres with $\alpha$-element enhanced compositions $([\alpha / \mathrm{Fe}]=+0.4$; Castelli \& Kurucz $2003)$ in order to set the $T_{\text {eff }}$ for $\alpha$ Boo. The flux from $\lambda$ $\sim 0.3-10 \mu \mathrm{m}$ was used with the resultant best-fit $T_{\text {eff }}=4286 \pm$ $30 \mathrm{~K}$. This value is entirely consistent with that obtained here $\left(T_{\text {eff }}=4275 \pm 50 \mathrm{~K}\right)$ based on the $(J-K)$ color and calibration used here from Bessell et al. (1998) and Gonzalez Hernandez \& Bonifacio (2009).

Ramirez \& Allende Prieto (2011) used stellar evolutionary model tracks to estimate the mass (as well as age) of $\alpha$ Boo, as the effective temperature and parallax are known to high precision. The mass was set by comparison to Yonsei-Yale isochrones (Yi et al. 2001; Kim et al. 2002) and was found to be $M=1.08 \pm$ $0.06 M_{\odot}$, which yields a surface gravity of $\log g=1.66 \pm$ 0.05 . In this study the $T_{\text {eff }}=4275 \mathrm{~K}$ value from the $(J-K)$ color is adopted, while the Ramirez \& Allende Prieto $\log g$ is used. These stellar parameters were used to generate a model atmosphere as described in Section 4 with the ATLAS9 code in the same manner as is being done for the APOGEE targets (Meszaros et al. 2012).

The microturbulence, $\xi$, derived by Ramirez \& Allende Prieto was set by $\mathrm{Fe}$ I lines and was found to be $\xi=1.74 \mathrm{~km} \mathrm{~s}^{-1}$. The Fe I lines used from the APOGEE linelist yielded a value of $\xi=$ $1.85 \pm 0.05 \mathrm{~km} \mathrm{~s}^{-1}$ : very close to that derived from optical Fe I lines.

A direct comparison of the derived abundances here with those from Ramirez \& Allende Prieto (2011) using completely different sets of lines leads to the following mean difference and standard deviation for 13 elements in common of $\Delta A(\mathrm{x})$ (this paper; Ramirez \& Allende Prieto) $=+0.03 \pm 0.10$ dex. There is no significant offset in the overall abundance patterns and the scatter is that expected given the uncertainties in the log $g f$ scales and internal line-to-line scatter within each study. This comparison suggests that abundances derived from the APOGEE linelist can be used for comparisons to those found from analyses at visual wavelengths when high-quality spectra are analyzed.

$$
\text { 4.5.2. } \mu \text { Leo }
$$

This K-giant is often cited as the "prototype" for metal-rich stars, having been noted as such by Spinrad \& Taylor (1969), and some of its spectral regions contain numerous and strong $\mathrm{CN}$ lines. Two older abundance studies in the literature (Gratton \& Sneden 1990; Smith \& Ruck 2000) are used to compare to the abundances derived here, as well as a more recent analysis by Fulbright et al. (2007; FMR). As with the $\alpha$ Boo comparison in Section 4.5.1, both of these published analyses are based on high-resolution spectra in the visual. Both studies used $T_{\text {eff }}=$ $4540 \mathrm{~K}$ for $\mu$ Leo, which is similar to the value $4550 \mathrm{~K}$ adopted here. The surface gravities were slightly different, with $\log g=$ $2.3 \pm 0.3$ in Gratton \& Sneden and $2.2 \pm 0.1$ in Smith \& Ruck; both values agree with the value here, within the uncertainties, of $\log g=2.1$ derived from the luminosity and estimate of stellar mass.

The value of $\xi=1.8 \mathrm{~km} \mathrm{~s}^{-1}$ found from the $H$-band Fe I lines is slightly higher than $1.2 \mathrm{~km} \mathrm{~s}^{1}$ found by both Gratton \& Sneden (1990) and Smith \& Ruck (2000), but with all studies having uncertainties of $0.3-0.5 \mathrm{~km} \mathrm{~s}^{-1}$, this offset cannot be claimed to be significant. A comparison of abundances between here and Gratton \& Sneden (1990) for 12 elements finds a mean $\Delta A(\mathrm{x})$ (this paper; GS) $=-0.05 \pm 0.14 \mathrm{dex}$, while the same comparison with Smith \& Ruck (2000) yields $\Delta A(\mathrm{x})=-0.05 \pm$ 0.02 dex for the three elements analyzed by them: Fe, $\mathrm{Mg}$, and $\mathrm{Ca}$. The abundance offsets are not significant and the scatter is within what is expected.

The more recent abundance analysis of $\mu$ Leo by FMR was part of their study of bulge red giants. Their derived stellar parameters were quite similar to those found here, with $T_{\text {eff }}(\mathrm{FMR})=4520 \mathrm{~K},(\Delta T$ (this study; FMR $\left.)=+30 \mathrm{~K}\right), \log g=$ $2.33(\Delta \log g$ (this study; FMR $)=-0.23)$, and $\xi=$ $1.50 \mathrm{~km} \mathrm{~s}^{-1}(\Delta \xi$ (this study; FMR $\left.)=+0.3 \mathrm{~km} \mathrm{~s}^{-1}\right)$. There are seven elements in common ( $\mathrm{Fe}, \mathrm{O}, \mathrm{Mg}, \mathrm{Al}, \mathrm{Si}, \mathrm{Ca}$, and $\mathrm{Ti}$ ) and the mean difference is $\Delta A(\mathrm{x})$ (this study; FMR) 
$=-0.10 \pm 0.13$ dex. A comparison among the three $\mu$ Leo studies points to rather small offsets in stellar parameters $(\sim 30 \mathrm{~K}$ in $T_{\text {eff }}, 0.2$ dex in $\log g, 0.3-0.5 \mathrm{~km} \mathrm{~s}^{-1}$ in $\xi$, and $0.1 \mathrm{dex}$ in $\left.[\mathrm{m} / \mathrm{H}]\right)$ with the conclusion that $H$-band spectroscopy can be compared reliably to optical analyses with reasonable accuracies, even at this higher metallicity.

\subsection{3. $\beta$ And}

This M-giant was analyzed previously by Smith \& Lambert (1985) who derived $T_{\text {eff }}=3800 \mathrm{~K}$ (from its $(V-K)$ color), $\log g=1.6$, and $\xi=2.1 \mathrm{~km} \mathrm{~s}^{-1}$. The derived effective temperature here of $3825 \mathrm{~K}$ is very close to Smith \& Lambert, as is the microturbulence of $2.2 \mathrm{~km} \mathrm{~s}^{-1}$. The surface gravity from Smith \& Lambert is larger by 0.7 dex, but their value relied on the distance (as it does in this paper), for which they used a luminosity calibration for the Ca II K-line reversal that was tied to a pre-Hipparcos Hyades distance that was too small. Making the estimated log $g$ corrections to the Smith \& Lambert (1985) abundances (based on their published sensitivities of abundance with $\log g$ ) for ${ }^{12} \mathrm{C},{ }^{14} \mathrm{~N},{ }^{16} \mathrm{O}, \mathrm{Ti}, \mathrm{Fe}$, and $\mathrm{Ni}$ we find a mean difference and standard deviation of $\Delta A(\mathrm{x})$ (this paper; Smith $\&$ Lambert $)=+0.02 \pm 0.14$ dex .

Beta And was also used as a comparison star by Chou et al. (2010) in their study of Sgr dwarf-galaxy tidal streams in the Galactic halo. Chou et al. (2010) applied a similar technique as that employed here to estimate temperatures and gravities and found $T_{\text {eff }}=3850 \pm 75 \mathrm{~K}, \log g=0.9$, and $\xi=1.96 \mathrm{~km} \mathrm{~s}^{-1}$, giving differences of only $25 \mathrm{~K}$ in $T_{\text {eff }}, 0.0$ in $\log g$, and $+0.24 \mathrm{~km} \mathrm{~s}^{-1}$ in $\xi$. Their analysis used the spectral region around $7500 \AA$ containing a sample of $\mathrm{Fe}_{\mathrm{I}}$ and $\mathrm{Ti}$ I lines and determined $A(\mathrm{Fe})=7.12 \pm 0.06$ and $A(\mathrm{Ti})=4.55 \pm 0.12$, which can be compared to values of $7.23 \pm 0.03$ and $4.72 \pm 0.02$, respectively, here, yielding differences of +0.11 dex in $\mathrm{Fe}$ and +0.17 dex in Ti.

\subsubsection{HD 199799}

HD 199799 is classified as spectral type M2S, which means that it displayed enhanced bands of $\mathrm{ZrO}$ in classification spectra. Smith \& Lambert (1988) detected the radioactive $s$-process element Tc I in its spectrum, identifying this star as an intrinsic thermally pulsing asymptotic giant branch (TP-AGB) star undergoing third dredge-up. A quantitative abundance analysis was then carried out by Smith \& Lambert (1990) and they derived similar stellar parameters to those derived here: the same $T_{\text {eff }}=3400 \mathrm{~K}$, with a slightly lower value of $\log g=+0.3$, compared to +0.5 here. The microturbulent velocity determined here from the $H$-band spectra is $\xi=2.4 \mathrm{~km} \mathrm{~s}^{-1}$, which is $0.7 \mathrm{~km} \mathrm{~s}^{-1}$ larger than Smith \& Lambert (1990), who used Fe I lines near $7500 \AA$. Taking into account the slightly lower gravity and smaller $\xi$, the abundances from Smith \& Lambert (1990) can be compared to those here for $\mathrm{Fe},{ }^{12} \mathrm{C},{ }^{14} \mathrm{~N},{ }^{16} \mathrm{O}, \mathrm{Ti}, \mathrm{Cr}, \mathrm{Co}$, and $\mathrm{Ni}$, with the mean difference being $\Delta A(\mathrm{x})(\mathrm{Us}-\mathrm{SL90})=$ $-0.08 \pm 0.13$. The respective carbon isotopic ratios compare well, with Smith \& Lambert (1990) finding ${ }^{12} \mathrm{C} /{ }^{13} \mathrm{C}=28$, while a value of 27 is obtained here. The derived $\mathrm{C} / \mathrm{O}$ ratios are the same in the two studies, with ${ }^{12} \mathrm{C} /{ }^{16} \mathrm{O}=0.68$.

The comparisons presented here are between studies that have possibly used somewhat different families of model atmospheres (e.g., MARCS or ATLAS), different spectral regions, and linelists derived from heterogeneous sources. Given the variety of data and analysis techniques, the derived abundances, in all cases, compare well at approximately the 0.10-0.15 dex level. A glance at Table 7 reveals that such differences fall within this range of uncertainty for most elements given modest changes in fundamental model atmosphere parameters. Since the derived stellar parameters themselves rely to some degree on the type of analysis, the generally good agreement between the derived abundances from the various published sources, along with the expected uncertainties in this study, as presented in Table 7, is encouraging. The gist of the comparisons, since many of the studies are based on optical spectra, is that $H$-band spectroscopy can be used to define a precise internal abundance scale that can also be extended to abundances derived from other, more common spectral windows, such as in the optical.

\section{DISCUSSION}

The abundances from Table 6 and an estimation of their respective uncertainties from Table 7 , along with the stellar parameters from Table 2 represent, to some extent, what ASPCAP will produce from the APOGEE spectra. The following discussion highlights these abundances in light of internal red giant stellar evolution, as well as Galactic chemical evolution and stellar populations. These discussion topics anticipate what sorts of information will be gleaned from the APOGEE targets.

\subsection{CNO Abundances and Red Giant Evolution}

Based on their luminosities and effective temperatures (Figure 2), four of the red giants studied here are either firstascent giants or, perhaps for $\mu$ Leo, a clump red giant. HD 199799 is in a more advanced stage of stellar evolution where it is undergoing thermal pulses and third dredge-up on the AGB. The combination of nucleosynthesis and convective mixing in evolved red giants should result in surface abundances of ${ }^{12} \mathrm{C}$, ${ }^{13} \mathrm{C}$, and ${ }^{14} \mathrm{~N}$ that have been altered from their initial (main sequence) values due to $\mathrm{CN}$-cycle $\mathrm{H}$-burning and first dredge-up.

The result of $\mathrm{CN}$-cycle mixing is seen mostly easily in the ${ }^{12} \mathrm{C} /{ }^{13} \mathrm{C}$ ratios which are much higher in main-sequence stars, with the Sun having a value of 89 . All four of the RGB or clump giants have quite low values, ranging from ${ }^{12} \mathrm{C} /{ }^{13} \mathrm{C} \sim 6-20$. Gilroy (1989) and many subsequent studies of both open and globular clusters (e.g., Mikolaitis et al. 2012 or Briley et al. 1995) have noted that the carbon isotopic ratios correlate with stellar mass in stars above the luminosity bump on the RGB or in the clump; red giants more massive than $M \sim 2.5 M_{\odot}$ have values of ${ }^{12} \mathrm{C} /{ }^{13} \mathrm{C} \sim 22-30$, while lower-mass red giants exhibit decreasing isotopic ratios with declining mass. The mass estimates derived here for field stars from distance-luminosity and stellar evolutionary tracks are modestly well defined and the top panel of Figure 5 shows the ${ }^{12} \mathrm{C} /{ }^{13} \mathrm{C}$ values versus the estimated stellar mass. Also plotted are representative results for open clusters from Gilroy (1989) and Mikolaitis et al. (2012), and the globular cluster M71 from Briley et al. (1995). The cluster red giants have recently evolved from the cluster turnoffs, which have a well-determined mass. For the lower-mass stars included in these cluster studies, evolution along the RGB is rapid $\left(\sim 10^{7} \mathrm{yr}\right)$ when compared to main-sequence lifetimes $\left(\sim 10^{8}-10^{9}\right.$ yr) so the red giant mass is similar to the turn-off mass. This can be demonstrated using the evolutionary tracks from Bertelli et al. (2009), where an isochrone with a turn-off mass of $4.0 M_{\odot}$ has a red giant tip mass of $4.2 M_{\odot}$, or a ratio of RGB to turn-off (TO) masses of 1.05. This ratio of masses becomes even closer to 1.0 for lower masses, which include all of the points in Figure 5. The points from Gilroy represent the mean values of ${ }^{12} \mathrm{C} /{ }^{13} \mathrm{C}$ for 19 open clusters with a total of 55 red giants in her sample. The mean carbon isotopic ratios 


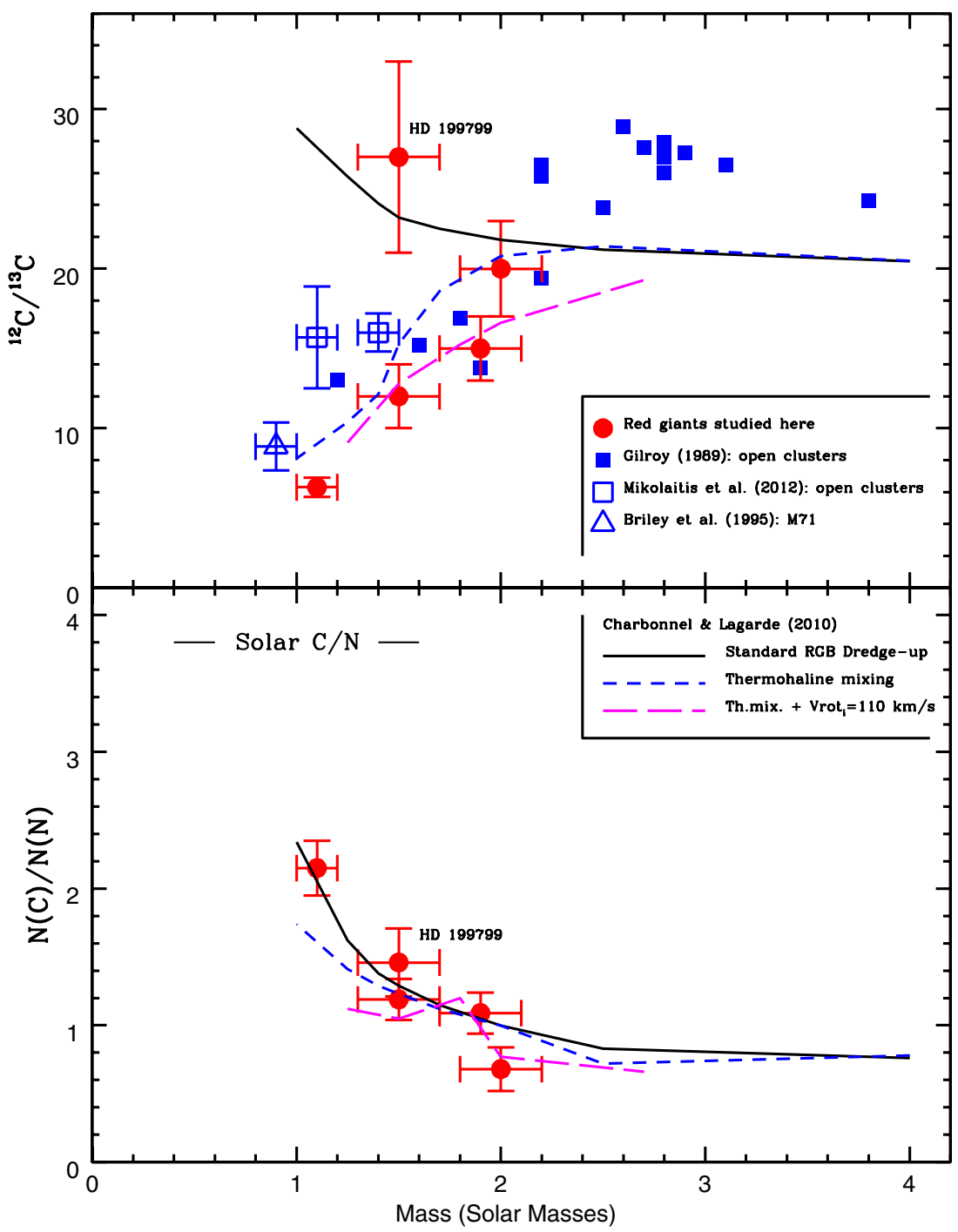

Figure 5. Behavior of the nuclei ${ }^{12} \mathrm{C},{ }^{13} \mathrm{C}$, and ${ }^{14} \mathrm{~N}$ with stellar mass on the RGB as characterized by ${ }^{12} \mathrm{C} /{ }^{13} \mathrm{C}$ and $\mathrm{C} / \mathrm{N}\left(\right.$ where $\mathrm{C}={ }^{12} \mathrm{C}+{ }^{13} \mathrm{C}$ and $\left.\mathrm{N}={ }^{14} \mathrm{~N}\right)$. The relative abundances of these nuclei are altered by H-burning via the $\mathrm{CN}$-cycle and convective mixing on the RGB dredges up the processed abundances. The TP-AGB star HD 199799 is identified, as it has significantly increased its ${ }^{12} \mathrm{C}$ abundance from He-burning and third dredge-up and has thus increased its ${ }^{12} \mathrm{C} /{ }^{13} \mathrm{C}$ relative to when it was on the RGB.

(A color version of this figure is available in the online journal.)

for two other open clusters (with a total of 10 red giants from Cr261 or NGC 6253) from Mikolaitis et al. (2012) are also shown (open blue squares). A representative globular cluster, M71, which has a lower main-sequence mass $\left(M \sim 0.9 M_{\odot}\right)$ from Briley et al. (1995) is plotted as the open blue triangle and represents the mean of two of the $\mathrm{CN}$-weak stars in this cluster. The $\mathrm{CN}$-strong giants that Briley et al. also studied are not plotted, as they exhibit slightly lower isotopic ratios but almost certainly represent second-generation stars in M71 that were formed from large fractions of the ejecta of first-generation intermediate-mass red giants and thus are not representative of single-star red giant evolution.

The curves in the top panel of Figure 5 represent stellar evolutionary model results from Charbonnel \& Lagarde (2010); the solid black line illustrates their predictions for standard RGB dredge-up (no "extra mixing" or rotation included). Clearly, standard RGB first dredge-up does not fit the observed trend of ${ }^{12} \mathrm{C} /{ }^{13} \mathrm{C}$ for red giants with $M \leqslant 2.2 M_{\odot}$. This poor comparison between theory and observation has led to work in trying to identify other types of "extra mixing" mechanisms that would lead to lower carbon isotopic ratios in the lower-mass red giants. Two such hypothesized mechanisms are thermohaline mixing or thermohaline mixing coupled with rotational mixing, as discussed recently by Charbonnel \& Lagarde (2010). The dashed curves present results from Charbonnel \& Lagarde also, with the short dashes for thermohaline mixing only and the long dashes for thermohaline mixing plus rotational mixing for models with initial equatorial rotational velocities of $110 \mathrm{~km} \mathrm{~s}^{-1}$. The stellar models that incorporate these particular types of extra mixing provide fair agreement with the observations, although these are not the only types of processes that have been studied and are used here to merely illustrate the effect of extra mixing. The trends found in the clusters also overlap well with the field stars analyzed here, except for HD 199799, which is in a different evolutionary phase than the other red giants. This star has enriched its surface in ${ }^{12} \mathrm{C}$ that was synthesized by He-burning as a TP-AGB star. In the simplest case of pure ${ }^{12} \mathrm{C}$ dredge-up, the surface ${ }^{12} \mathrm{C} /{ }^{13} \mathrm{C}$ ratio would increase, which is where HD 199799 lies in the top panel of Figure 5 relative to the other red giants. Such an effect has been noted by both Lambert 


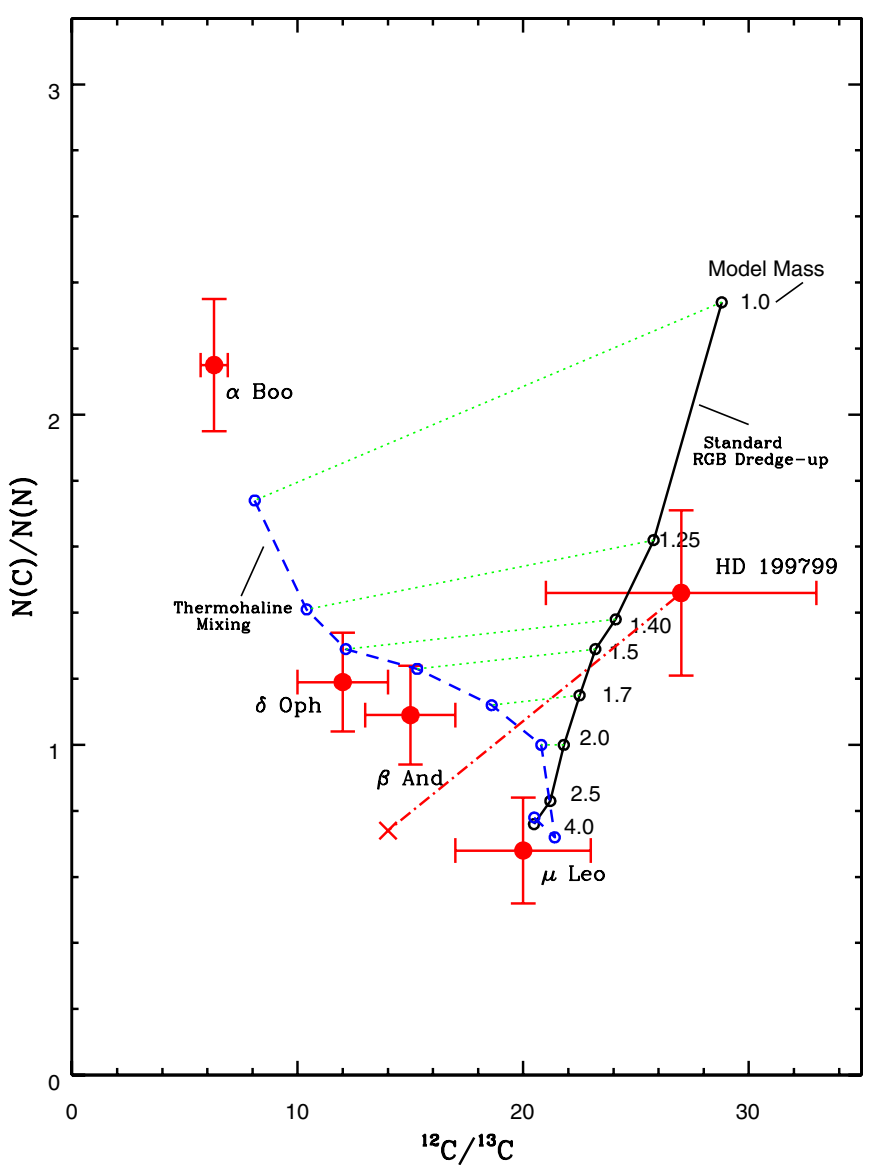

Figure 6. Observed and model predicted behavior of $N(\mathrm{C}) / N(\mathrm{~N})$ vs. ${ }^{12} \mathrm{C} /{ }^{13} \mathrm{C}$; the model curves show the so-called standard dredge-up (solid line curve) and a thermohaline mixing model as a function of stellar mass from Charbonnel \& Lagarde (2010; dashed line curve). Standard dredge-up does not predict the observed behavior of $N(\mathrm{C}) / N(\mathrm{~N})$ as a function of ${ }^{12} \mathrm{C} /{ }^{13} \mathrm{C}$ and some type of additional mixing process is needed. The TP-AGB star HD 199799 is identified here as it has dredged-up enough ${ }^{12} \mathrm{C}$ to have approximately doubled its abundance relative to its abundance on the RGB; the dash-dotted line moves HD 199799 to its approximate position when it was evolving along the RGB.

(A color version of this figure is available in the online journal.)

et al. (1986) and Smith \& Lambert (1990), where these studies find increasing values of ${ }^{12} \mathrm{C} /{ }^{13} \mathrm{C}$ as TP-AGB stars evolve from $\mathrm{M}$ to $\mathrm{MS}$ to $\mathrm{S}$ to $\mathrm{C}$ stars (with increasing values of ${ }^{12} \mathrm{C} /{ }^{16} \mathrm{O}$ ).

The bottom panel of Figure 5 plots the abundance by number ratio of $N(\mathrm{C}) / N(\mathrm{~N})$ versus stellar mass, where the carbon abundance is now the total of ${ }^{12} \mathrm{C}+{ }^{13} \mathrm{C}$ : for reference, the solar ratio is 3.80. Nitrogen is also the total abundance, however, in the case of the observations, ${ }^{15} \mathrm{~N}$ is ignored as its abundance is very low (and not detected in these red giants) when compared to ${ }^{14} \mathrm{~N}$. There is an observed decrease in $\mathrm{C} / \mathrm{N}$ with increasing mass as predicted by both standard RGB dredge-up and dredgeup plus thermohaline mixing (Charbonnel \& Lagarde 2010), which are shown as the solid and dashed curves, respectively, and are from the same models as in the top panel. In the case of the $\mathrm{C} / \mathrm{N}$ ratios, the total carbon and nitrogen abundances are not as strongly influenced by non-standard mixing as the carbon isotopic ratios.

Figure 6 combines the $\mathrm{C} / \mathrm{N}$ ratios versus ${ }^{12} \mathrm{C} /{ }^{13} \mathrm{C}$ ratios for both the observed red giants and the models from Charbonnel \& Lagarde (2010). When displayed this way, the different behavior between $\mathrm{C} / \mathrm{N}$ versus ${ }^{12} \mathrm{C} /{ }^{13} \mathrm{C}$ is striking and these two values are purely spectroscopically derived quantities. The trend of

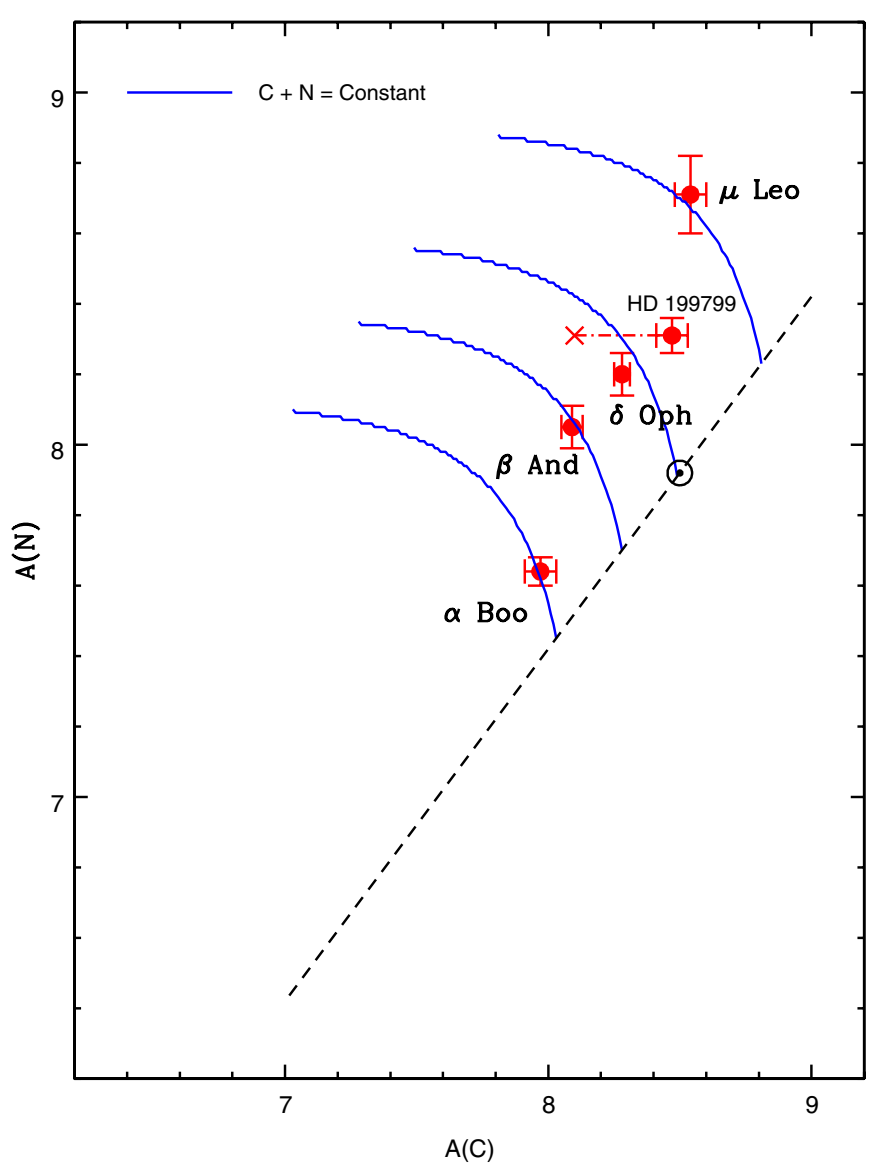

Figure 7. Testing if total $N(\mathrm{C})+N(\mathrm{~N})$ is conserved, as would be expected for pure $\mathrm{CN}$-cycle dredge-up. The dashed line represents metallicity scaled values of $A(\mathrm{C})$ and $A(\mathrm{~N})$ with a solar $\mathrm{C} / \mathrm{N}$ ratio. The blue curves map constant values of $\mathrm{C}+\mathrm{N}$ scaled to the metallicity (taken to be $[\mathrm{Fe} / \mathrm{H}]$ ) of each star. Clearly, initial (main-sequence) values of $[\mathrm{C}+\mathrm{N} / \mathrm{Fe}]=0$, followed by simple $\mathrm{CN}$-cycle dredgeup, are adequate representations of what is observed in these field RGB stars. The TP-AGB star HD 199799 has increased it ${ }^{12} \mathrm{C}$ abundance by approximately 0.35 dex and this shift in $A(C)$ is shown by the dash-dotted line.

(A color version of this figure is available in the online journal.)

$\mathrm{C} / \mathrm{N}$ with ${ }^{12} \mathrm{C} /{ }^{13}$ seems to provide a sensitive indicator of red giant mixing processes, as well as yields a mass estimate for stars on the RGB. Again, HD 199799 stands apart from the other red giants as it has internally enhanced its ${ }^{12} \mathrm{C}$ abundance by about a factor of two since it was an RGB star, based on its ${ }^{12} \mathrm{C} /{ }^{13} \mathrm{C}$ and mass, relative to other RGB stars in the top panel of Figure 5. The dash-dotted line connects to the region in this diagram where HD 199799 would fall if its surface ${ }^{12} \mathrm{C}$ abundance were reduced by a factor of two, which is a simplified sketch of how TP-AGB stars might evolve across such a diagram from the RGB.

The assumption of only $\mathrm{CN}$-cycle mixing taking place in the red giants is tested in Figure 7, where the total carbon abundance $\left({ }^{12} \mathrm{C}+{ }^{13} \mathrm{C}\right)$ is plotted versus the nitrogen abundance (here $\left.{ }^{14} \mathrm{~N}\right)$. The solar values of $\mathrm{C}$ and $\mathrm{N}$ are also shown, along with the dashed line illustrating scaled solar abundances for both $\mathrm{C}$ and $\mathrm{N}$. The continuous blue curves represent decreasing carbon and increasing nitrogen abundances from the scaled solar lines, with the constraint that the number of $\mathrm{C}+\mathrm{N}$ nuclei is conserved, as in the $\mathrm{CN}$-cycle (with no leakage into the $\mathrm{ON}$-cycle). Each curve is scaled in its initial $\mathrm{C}$ and $\mathrm{N}$ abundances by the stellar value of $[\mathrm{Fe} / \mathrm{H}]$ for $\alpha$ Boo, $\beta$ And, $\delta$ Oph, and $\mu$ Leo. CN-cycle mixing alone is sufficient to represent the derived abundances of $\mathrm{C}$ and 


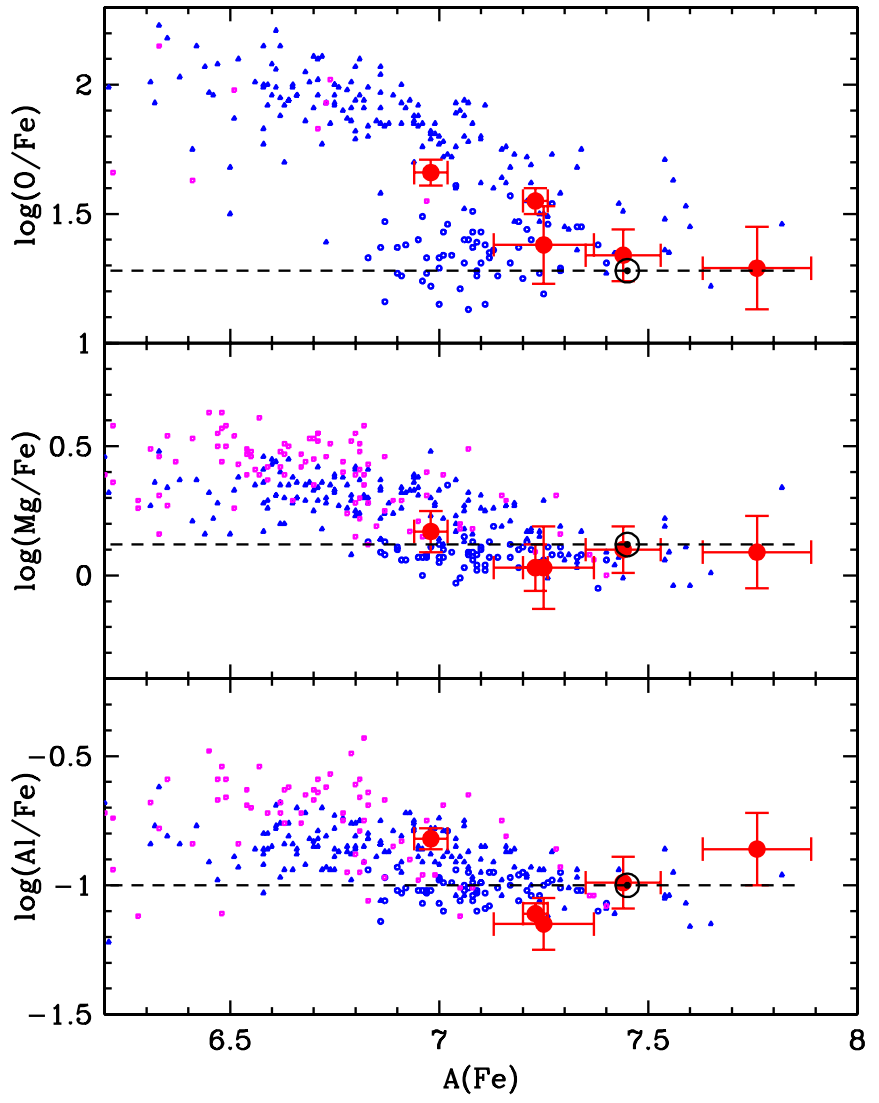

Figure 8. Abundances of oxygen, magnesium, and aluminum, relative to iron, shown as $\log (N(\mathrm{X}) / N(\mathrm{H}))$ vs. $A(\mathrm{Fe})$. The five stars studied here are represented by the filled red circles. The small blue symbols are results from Reddy et al. $(2003,2006)$ and the lower metallicity small magenta symbols are from Fulbright (2002) and Johnson (2002). The solar position is indicated with the dashed line showing the solar abundance ratio. The abundances derived here using the APOGEE linelist are well mapped into the general trends observed for Galactic field populations. The error bars are taken from the standard deviations in Table 6. Errors for the ratios are quadrature sums of the element in question and $\mathrm{Fe}$ I values of $\sigma$.

(A color version of this figure is available in the online journal.)

$\mathrm{N}$ with initial abundances having $[\mathrm{C}+\mathrm{N} / \mathrm{Fe}] \sim 0.0$ for each RGB star. Due to having undergone a third dredge-up and ${ }^{12} \mathrm{C}$ enrichment, HD 199799 will be offset from its CN curve, so no curve is plotted for it. The dash-dotted line simply shows the shift in $A(C)$ if HD 199799 has doubled its RGB ${ }^{12} \mathrm{C}$ abundance. Having nearly the same metallicity as $\beta$ And, HD 199799 might be expected to have been near the $\mathrm{CN}$ curve for $\beta$ And before it began its evolution as a TP-AGB star.

The APOGEE $H$-band wavelength region presents good opportunities for probing many nuclear and mixing processes that occur over the various phases of red giant evolution.

\subsection{Element Ratios and Galactic Chemical Evolution}

Other than the $\mathrm{C}$ and $\mathrm{N}$ isotopes, the vast majority of APOGEE red giant targets will not have altered their surface abundances of the other studied elements, so the remaining elements are dominated by chemical evolution within the various stellar populations of the Milky Way (or its accreted systems). The derived abundances of each of the other elements are discussed in the light of observed trends of these elements with $A(\mathrm{Fe})$ from other studies in the literature. The number of published abundances is now enormous; however, in this first exploratory study using the APOGEE linelist, a comparison with

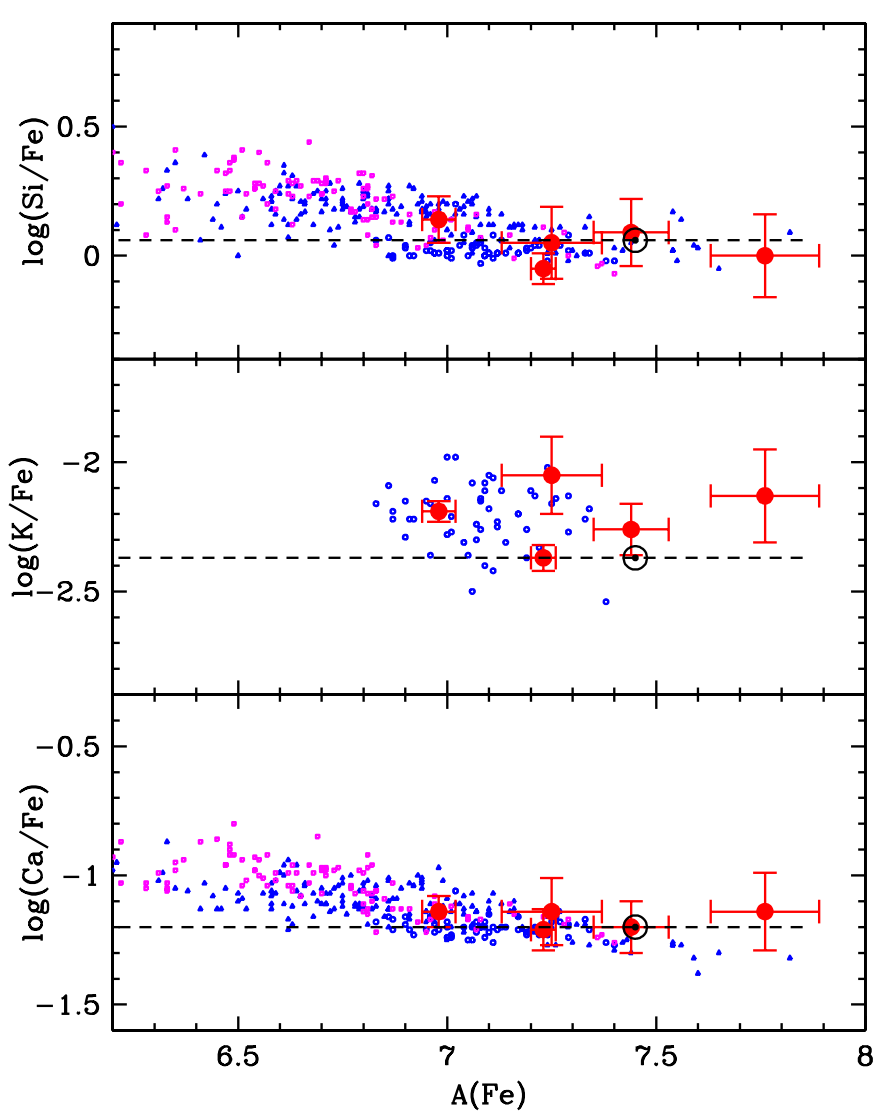

Figure 9. Abundances of silicon, potassium, and calcium, relative to iron, vs. $A(\mathrm{Fe})$. The plotting format and symbols are the same as in Figure 8.

(A color version of this figure is available in the online journal.)

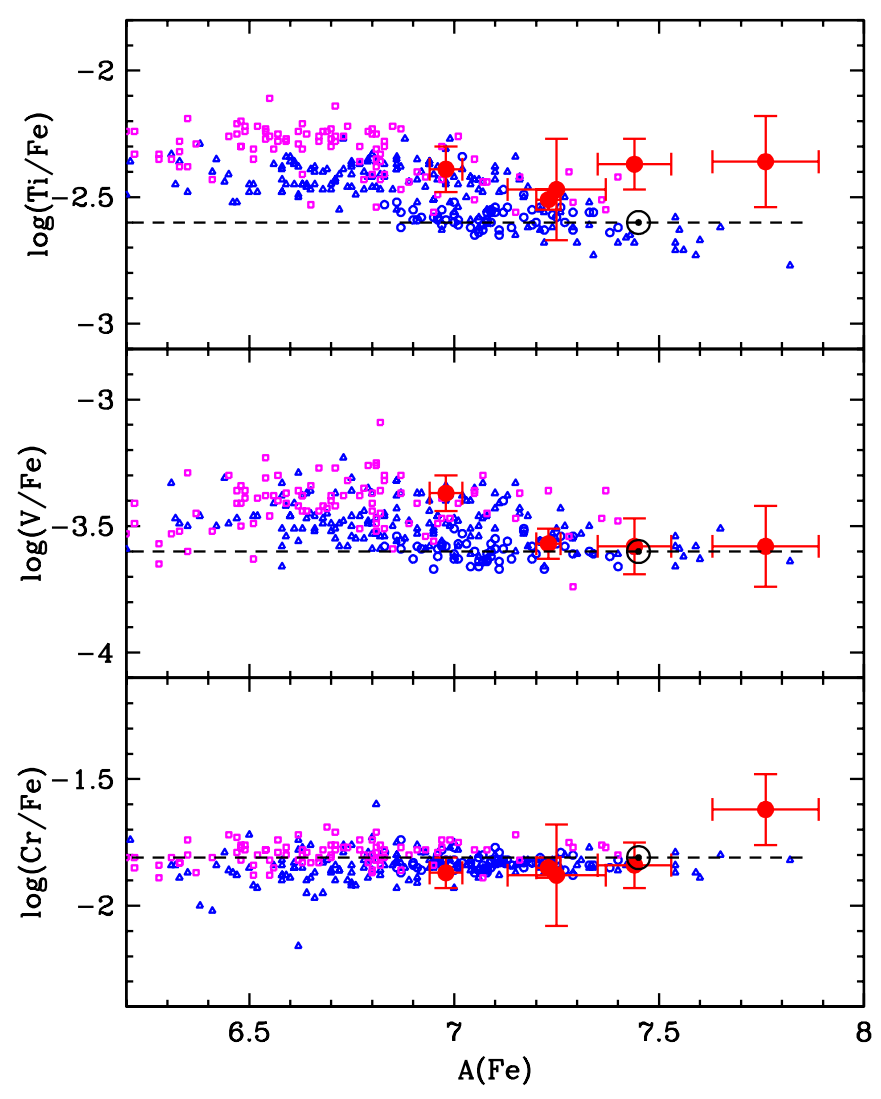

Figure 10. Abundances of titanium, vanadium, and chromium, relative to iron, vs. $A(\mathrm{Fe})$. The plotting format and symbols are the same as in Figure 8.

(A color version of this figure is available in the online journal.) 


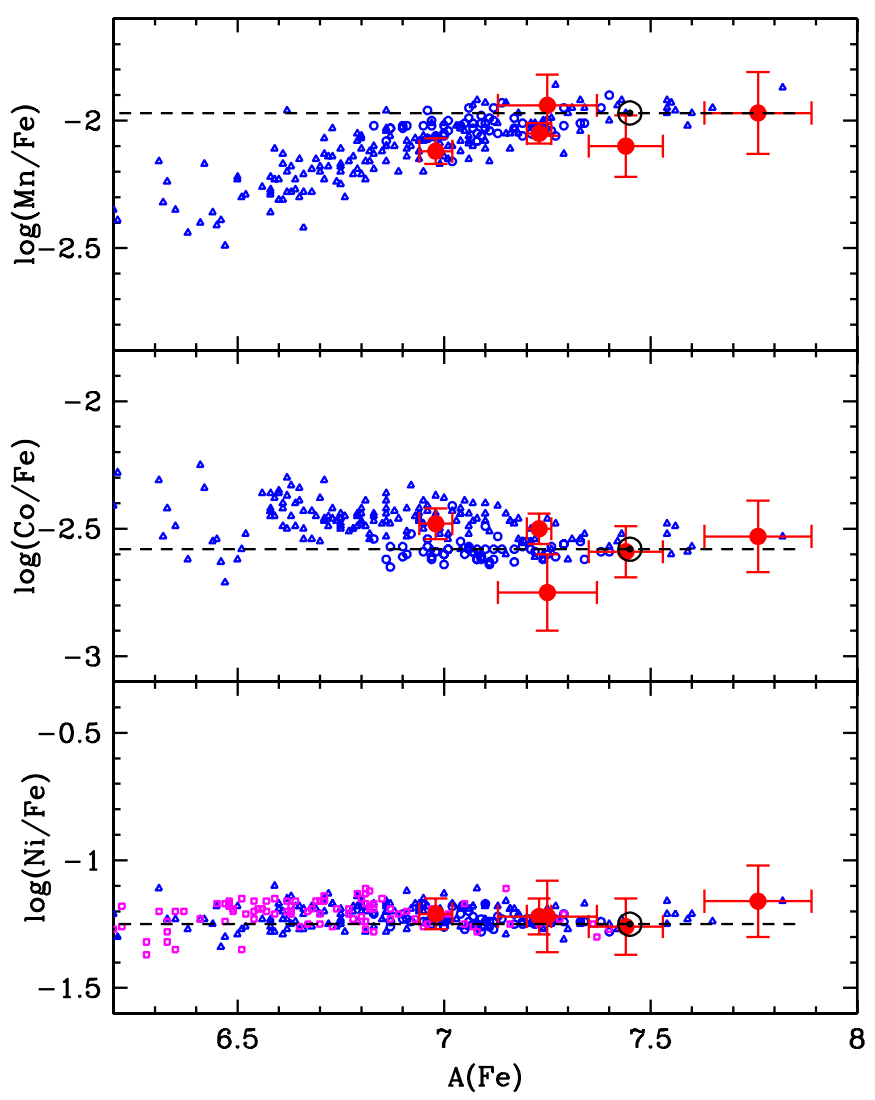

Figure 11. Abundances of manganese, cobalt, and nickel, relative to iron, vs. $A(\mathrm{Fe})$. The plotting format and symbols are the same as in Figure 8.

(A color version of this figure is available in the online journal.)

a small number of selected literature sources is sufficient. For comparison purposes, in this paper a stellar sample representative of the Milky Way stellar populations was constructed using Reddy et al. (2003; thin disk with some thick disk and having 58 stars), Reddy et al. (2006; thick disk with 176 stars), Johnson (2002; halo with 23 stars), and Fulbright (2002; halo with 178 stars).

The investigation of elements derived from the APOGEE linelist is via a number of figures, beginning with Figure 8 , which presents values of $\mathrm{O} / \mathrm{Fe}, \mathrm{Mg} / \mathrm{Fe}$, and $\mathrm{Al} / \mathrm{Fe}$ versus $A(\mathrm{Fe})$; the shorthand notation here of $\mathrm{x} / \mathrm{Fe}$ denotes the logarithmic number ratio of element $\mathrm{x}$ to iron, $\log [N(\mathrm{x}) / N(\mathrm{Fe})]$, or $A(\mathrm{x})-A(\mathrm{Fe})$. This nomenclature is chosen over the $[\mathrm{x} / \mathrm{Fe}]$ in the plots as it presents a direct logarithmic number ratio with the solar abundance ratio being noted in each panel, thus no reference to the underlying solar abundance is needed, but the relative scales are equivalent to $[\mathrm{x} / \mathrm{Fe}]$. The top panel of Figure 8 shows the behavior of $\mathrm{O} / \mathrm{Fe}$ and, as has been well known for decades, values of $\mathrm{O} / \mathrm{Fe}$ are elevated in the thick disk and halo stars. Among the field red giants here, the slightly metal-poor objects $\alpha$ Boo and $\beta$ And both have $\mathrm{O} / \mathrm{Fe}$ enhanced by $\sim+0.2-+0.3$. The near-solar metallicity M-giant $\delta$ Oph has a near-solar ratio of $\mathrm{O} / \mathrm{Fe}$, while the metal-rich giant $\mu$ Leo also exhibits a near-solar value $\mathrm{O} / \mathrm{Fe}$.

The behavior of $\mathrm{Mg} / \mathrm{Fe}$ is shown in the middle panel of Figure 8 and, like oxygen, $\mathrm{Mg} / \mathrm{Fe}$ ratios are typically elevated in the thick disk and halo populations. The four red giants closest to solar in $A(\mathrm{Fe})$ all exhibit basically solar $\mathrm{Mg} / \mathrm{Fe}$ ratios, with $\alpha$ Boo having a slight enhancement of $\sim+0.1$ dex, which overlaps the thick disk populations.

Aluminum abundances are plotted in the bottom panel of Figure 8 and the behavior of $\mathrm{Al} / \mathrm{Fe}$ versus $A(\mathrm{Fe})$ is not so well

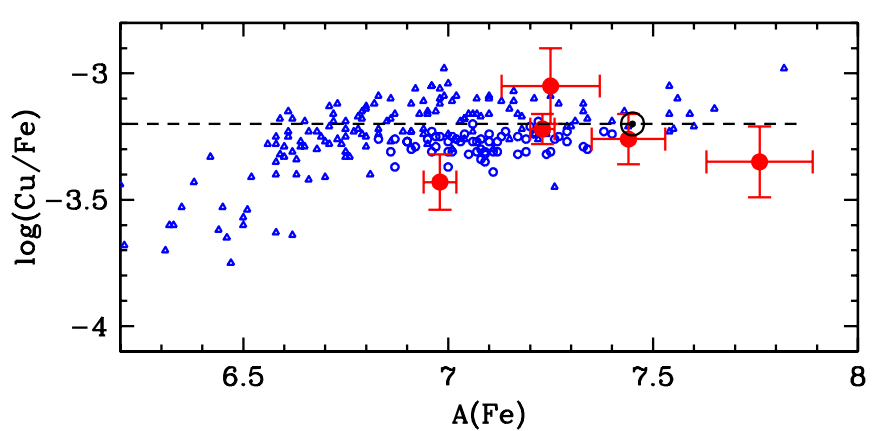

Figure 12. Abundance of copper relative to iron vs. $A(\mathrm{Fe})$. The plotting format and symbols are the same as in Figure 8.

(A color version of this figure is available in the online journal.)

defined, with relatively large scatter. Mu Leo is found to be modestly enhanced in $\mathrm{Al} / \mathrm{Fe}$ by about $+0.10-+0.15 \mathrm{dex}$, as is $\alpha$ Boo, with an enhancement of $\sim+0.2$.

The abundances for the rest of the elements studied here are illustrated in Figure 9 (for $\mathrm{Si}, \mathrm{K}$, and Ca), Figure 10 (for Ti, V, and $\mathrm{Cr}$ ), Figure 11 (for $\mathrm{Mn}, \mathrm{Co}$, and $\mathrm{Ni}$ ), and Figure 12 (for $\mathrm{Cu}$ ). In all elements, the general trends found in the general Galactic populations are recovered in the small sample of field red giants included here. The one exception is $\mathrm{Ti}$ in $\delta \mathrm{Oph}$ and $\mu$ Leo, both of which are found to exhibit enhanced values of $\mathrm{Ti} / \mathrm{Fe}$ of +0.2 dex. There is no obvious explanation for this, however, the continuing analysis of APOGEE spectra with this linelist will reveal if this possible effect appears in significant numbers of other red giants. The results presented and discussed here indicate that $H$-band spectra analyzed in LTE with the APOGEE linelist and 1-D model atmospheres can provide accurate results for chemical abundances.

\section{CONCLUSIONS}

Archival high-resolution FTS spectra from 15100 to $16900 \AA$ are analyzed to derive detailed chemical abundance distributions for five field red giants using the APOGEE linelist and model atmosphere grid. The red giants span a range in $T_{\text {eff }}$ from $3400 \mathrm{~K}$ to $4540 \mathrm{~K}$ and $\log g$ from 0.5 to 2.1 , covering a significant part of the effective temperature-surface gravity range being observed in the APOGEE survey.

Chemical abundances are determined for 16 elements, along with the minor carbon isotope, ${ }^{13} \mathrm{C}$. Comparisons of the abundances derived here with those from previously published studies of four of the target stars here, mostly from visual-wavelength high-resolution spectra, find abundance differences of $\sim 0.1$ dex. The APOGEE wavelength window with its derived linelist and model atmosphere grid, coupled to spectrum synthesis modeling, can be used to analyze high-resolution $H$-band spectra of red giants to probe both Galactic chemical evolution in stellar populations, as well as internal red giant nucleosynthesis and mixing.

We thank Ken Hinkle for helping to make the archival FTS spectral available and to Luan Ghezzi for useful comments on an initial draft of this paper. V.S. acknowledges partial support for this research from the National Science Foundation (AST1109888) and CAPES, Brazil. This publication makes use of data products from the Two Micron All Sky Survey, which is a joint project of the University of Massachusetts and the Infrared Processing and Analysis Center/California Institute of Technology, funded by the National Aeronautics 
and Space Administration and the National Science Foundation. This research has made use of the SIMBAD database, operated at CDS, Strasbourg, France.

Funding for SDSS-III has been provided by the Alfred P. Sloan Foundation, the Participating Institutions, the National Science Foundation, and the U.S. Department of Energy Office of Science. The SDSS-III Web site is http://www.sdss3.org/.

SDSS-III is managed by the Astrophysical Research Consortium for the Participating Institutions of the SDSS-III Collaboration including the University of Arizona, the Brazilian Participation Group, BrookhavenNational Laboratory, University of Cambridge, Carnegie Mellon University, University of Florida, the French Participation Group, the German Participation Group, Harvard University, the Instituto de Astrofisica de Canarias, the Michigan State/Notre Dame/JINA Participation Group, Johns Hopkins University, Lawrence Berkeley National Laboratory, Max Planck Institute for Astrophysics, New Mexico State University, New York University, Ohio State University, Pennsylvania State University, University of Portsmouth, Princeton University, the Spanish Participation Group, University of Tokyo, University of Utah, Vanderbilt University, University of Virginia, University of Washington, and Yale University.

Facility: Mayall (FTS)

\section{REFERENCES}

Bertelli, G., Girardi, L., Marigo, P., \& Nasi, E. 2008, A\&A, 484, 815 Bertelli, G., Nasi, E., Girardi, L., \& Marigo, P. 2009, A\&A, 508, 355 Bessell, M. S., Castelli, F., \& Plez, B. 1998, A\&A, 333, 231

Briley, M. M., Smith, V. V., \& Lambert, D. L. 1995, ApJL, 424, L119

Carpenter, J. M. 2001, AJ, 121, 285

Castelli, F., \& Kurucz, R. L. 2003, in Modelling of Stellar Atmospheres, Poster Contributions, IAU Symposium, Vol-210, ed. N. Piskunov, W. W. Weiss, \& D. F. Gray (San Francisco, CA: ASP), A20

Charbonnel, C., \& Lagarde, N. 2010, A\&A, 522, 10

Chou, M.-Y., Majewski, S. R., Cunha, K., et al. 2010, ApJL, 720, L5
Eisenstein, D. J., Weinberg, D. H., Agol, E., et al. 2011, AJ, 142, 72

Fulbright, J. P. 2002, AJ, 123, 404

Fulbright, J. P., McWilliam, A., \& Rich, R. M. 2007, ApJ, 661, 1152

Fusi Pecci, F., Ferraro, F. R., Crocker, D. A., Rood, R. T., \& Buonanno, R. 1990, A\&A, 238, 95

Gilroy, K. K. 1989, ApJ, 347, 835

Goldman, A., Shoenfeld, W. G., Goorvitch, D., et al. 1998, JQSRT, 59, 453

Gonzalez Hernandez, J. I., \& Bonifacio, P. 2009, A\&A, 497, 497

Goorvitch, D. 1994, ApJS, 95, 535

Gratton, R. G., \& Sneden, C. 1990, A\&A, 234, 366

Hall, D. N. B., Ridgway, S. T., Bell, E. A., \& Yarborough, J. M. 1979, Proc. SPIE, 172, 121

Hinkle, K., Wallace, L., \& Livingston, W. 1995, PASP, 107, 1042

Johnson, H. L., Mitchell, R. I., Iriarte, B., \& Wisniewski, W. Z. 1966, CoLPL, 4,99

Johnson, J. A. 2002, ApJS, 139, 219

Kim, Y., Demarque, P., Yi, S. K., \& Alexander, D. R. 2002, ApJS, 143, 499

Kurucz, R. L. 1993, ATLAS9 Stellar Atmosphere Programs and 2 km s1 Grid, Kurucz CD-ROM No. 13. (Cambridge, MA: Smithsonian Astrophysical Observatory), 13

Lambert, D. L., Gustafsson, B., Eriksson, K., \& Hinkle, K. H. 1986, ApJS, 62,373

Lodders, K. 2010, in Principles and Perspectives in Cosmochemistry, Astrophysics \& Space Science Proceedings, Part 3, ed. A. Goswami \& B. E. Reddy (Berlin: Springer), 379

Melendez, J., \& Barbuy, B. 1999, ApJS, 124, 527

Meszaros, S., Allende Prieto, C., Edvardsson, B., et al. 2012, AJ, 144, 120

Mikolaitis, S., Tautvaisiene, G., Gratton, R., Bragaglia, A., \& Carretta, E. 2012, A\&A, 541, 137

Ramirez, I., \& Allende Prieto, C. 2011, ApJ, 743, 135

Reddy, B. E., Lambert, D. L., \& Allende Prieto, C. 2006, MNRAS, 367, 1329

Reddy, B. E., Tomkin, J., Lambert, D. L., \& Allende Prieto, C. 2003, MNRAS, 340,304

Skrutskie, M. F., Cutri, R. M., Stiening, R., et al. 2006, AJ, 131, 1163

Smith, G., \& Ruck, M. J. 2000, A\&A, 356, 570

Smith, V. V., \& Lambert, D. L. 1985, ApJ, 294, 326

Smith, V. V., \& Lambert, D. L. 1988, ApJ, 333, 219

Smith, V. V., \& Lambert, D. L. 1990, ApJS, 72, 387

Sneden, C. 1973, ApJ, 184, 839

Spinrad, H., \& Taylor, B. J. 1969, ApJ, 157, 1279

van Leeuwen, F. 2007, A\&A, 474, 653

Yi, S., Demarque, P., Kim, Y., et al. 2001, ApJS, 136, 417 